\title{
The Concentration-compactness/ Rigidity Method for Critical Dispersive and Wave Equations
}

\author{
Carlos E. Kenig ${ }^{1}$
}

${ }^{1}$ Supported in part by NSF 
In these lectures I will describe a program (which I will call the concentrationcompactness/rigidity method) that Frank Merle and I have been developing to study critical evolution problems. The issues studied center around global wellposedness and scattering. The method applies to non-linear dispersive and wave equations in both defocusing and focusing cases. The method can be divided into two parts. The first part ("the concentration-compactness" part) is in some sense "universal" and works in similar ways for "all" critical problems. The second part ("the rigidity" part) has a "universal" formulation, but needs to be established individually for each problem. The method is inspired by the elliptic work on the Yamabe problem and by works of Merle, Martel-Merle and Merle-Raphäel in the non-linear Schrödinger equation and generalized KdV equations.

To focus on the issues, let us first concentrate on the energy critical nonlinear Schrödinger equation (NLS) and the energy critical non-linear wave equation (NLW). We thus have:

$$
\begin{cases}i \partial_{t} u+\Delta u \pm|u|^{4 / N-2} u=0, & (x, t) \in \mathbb{R}^{N} \times \mathbb{R} \\ \left.u\right|_{t=0}=u_{0} \in \dot{H}^{1}\left(\mathbb{R}^{n}\right), & N \geq 3,\end{cases}
$$

and

$$
\begin{cases}\partial_{t}^{2} u-\triangle u= \pm|u|^{4 / N-2} u, & (x, t) \in \mathbb{R}^{N} \times \mathbb{R}, \\ \left.u\right|_{t=0}=u_{0} \in \dot{H}^{1}\left(\mathbb{R}^{n}\right), & \\ \left.\partial_{t} u\right|_{t=0}=u_{1} \in L^{2}\left(\mathbb{R}^{n}\right), & N \geq 3 .\end{cases}
$$

In both cases, the "-" sign corresponds to the defocusing case, while the "+" sign corresponds to the focusing case. For (1), if $u$ is a solution, so is $\frac{1}{\lambda^{N-2 / 2}} u\left(\frac{x}{\lambda}, \frac{t}{\lambda^{2}}\right)$. For $(2)$, if $u$ is a solution, so is $\frac{1}{\lambda^{N-2 / 2}} u\left(\frac{x}{\lambda}, \frac{t}{\lambda}\right)$. Both scalings leave invariant the energy spaces $\dot{H}^{1}, \dot{H}^{1} \times L^{2}$ respectively, and that is why they are called energy critical. The energy which is conserved in this problem is

$$
\begin{gathered}
E_{ \pm}\left(u_{0}\right)=\frac{1}{2} \int\left|\nabla u_{0}\right|^{2} \pm \frac{1}{2^{*}} \int\left|u_{0}\right|^{2^{*}} \\
E_{ \pm}\left(\left(u_{0}, u_{1}\right)\right)=\frac{1}{2} \int\left|\nabla u_{0}\right|^{2}+\frac{1}{2} \int\left|u_{1}\right|^{2} \pm \frac{1}{2^{*}} \int\left|u_{0}\right|^{2^{*}}
\end{gathered}
$$

where $\frac{1}{2^{*}}=\frac{1}{2}-\frac{1}{N}=\frac{N-2}{2 N}$. The "+" corresponds to the defocusing case while the "-" corresponds to the focusing case.

In both problems, the theory of the local Cauchy problem has been understood for a while (in the case of (1), through the work of Cazenave-Weissler [7], while in the case of (2) through the works of Pecher [37], Ginibre-Velo [14], Ginibre-Velo-Soffer [13], and many others, for instance [3], [20], [34], [41], etc.). These works show that, say for (1), for any $u_{0}$ with $\left\|u_{0}\right\|_{\dot{H}^{1}} \leq \delta$, there exists a 
unique solution of (1) defined for all time and the solution scatters, i.e., there exist $u_{0}^{+}, u_{0}^{-}$in $\dot{H}^{1}$ such that

$$
\lim _{t \rightarrow \pm \infty}\left\|u(t)-e^{i t \Delta} u_{0}^{ \pm}\right\|_{\dot{H}^{1}}=0 .
$$

A corresponding result holds for $(2)$. Moreover, given any initial data $u_{0}\left(\left(u_{0}, u_{1}\right)\right)$ in the energy space, there exist $T_{+}\left(u_{0}\right), T_{-}\left(u_{0}\right)$ such that there exists a unique solution in $\left(-T_{-}\left(u_{0}\right), T_{+}\left(u_{0}\right)\right)$ and the interval is maximal (for $(2),\left(-T_{-}\left(u_{0}, u_{1}\right)\right.$, $\left.T_{+}\left(u_{0}, u_{1}\right)\right)$ ). In both problems, there exists a crucial space-time norm (or "Strichartz norm"). For (1), on a time interval $I$, we define

$$
\|u\|_{S(I)}=\|u\|_{L_{I}^{2(N+2) / N-2} L_{x}^{2(N+2) / N-2},}
$$

while for (2) we have

$$
\|u\|_{S(I)}=\|u\|_{L_{I}^{2(N+1) / N-2} L_{x}^{2(N+1) / N-2} .} .
$$

This norm is crucial, say for $(1)$, because, if $T_{+}\left(u_{0}\right)<+\infty$, we must have

$$
\|u\|_{S\left(\left(0, T_{+}\left(u_{0}\right)\right)\right)}=+\infty ;
$$

moreover, if $T_{+}\left(u_{0}\right)=+\infty, u$ scatters at $+\infty$ if and only if $\|u\|_{S(0,+\infty)}<+\infty$. Similar results hold for (2). The question that attracted people's attention here is: What happens for large data? The question was first studied for (2) in the defocusing case, through works of Struwe [44] in the radial case, Grillakis [16], [17] in the general case, for the preservation of smoothness, and in the terms described here in the works of Shatah-Struwe [41], [42], Bahouri-Shatah [3], Bahouri-Gérard [2], Kapitansky [20], etc. The summary of these works is that (this was achieved in the early 90's), for any pair $\left(u_{0}, u_{1}\right) \in \dot{H}^{1} \times L^{2}$, in the defocusing case we have $T_{ \pm}\left(u_{0}, u_{1}\right)=+\infty$ and the solution scatters. The corresponding results for (1) in the defocusing case took much longer. The first result was established by Bourgain [4] in 1998, who established the analogous result for $u_{0}$ radial, $N=3,4$, with Grillakis [18] showing preservation of smoothness for $N=3$ and radial data. Tao extended these results to $N \geq 5, u_{0}$ radial [48]. Finally, Colliander-Kell-StaffilaniTakaoka-Tao proved this for $N=3$ and all data $u_{0}$ [8], with extensions to $N=4$ by Ryckman-Vişan [40] and to $N \geq 5$ by Vişan [54] in 2005 .

In the focusing case, these results do not hold. In fact, for (2) H. Levine [33] showed in 1974 that in the focusing case, if $\left(u_{0}, u_{1}\right) \in \dot{H}^{1} \times L^{2}, u_{0} \in L^{2}$ and $E\left(\left(u_{0}, u_{1}\right)\right)<0$, there is always a break-down in finite time, i.e., $T_{ \pm}\left(u_{0}, u_{1}\right)<\infty$. He showed this by an "obstruction" type of argument. Recently Krieger-SchlagTătaru [32] have constructed radial examples $(N=3)$, for which $T_{ \pm}\left(u_{0}, u_{1}\right)<\infty$. For (1) a classical argument due to Zakharov and Glassey [15], based on the virial identity, shows the same result as H. Levine's if $\int|x|^{2}\left|u_{0}\right|^{2}<\infty, E\left(u_{0}\right)<0$. Moreover, for both (1) and (2), in the focusing case we have the following static solution:

$$
W(x)=\left(1+\frac{|x|^{2}}{N(N-2)}\right)^{-(N-2) / 2} \in \dot{H}^{1}\left(\mathbb{R}^{N}\right),
$$


which solves the elliptic equation

$$
\triangle W+|W|^{4 / N-2} W=0 .
$$

Thus, scattering need not occur for solutions that exist globally in time. The solution $W$ plays an important role in the Yamabe problem (see [1] for instance) and it does so once more here. The results in which I am going to concentrate here are:

Theorem 1 (Kenig-Merle [25]). For the focusing energy critical (NLS), $3 \leq N \leq 6$, consider $u_{0} \in \dot{H}^{1}$ such that $E\left(u_{0}\right)<E(W), u_{0}$ radial. Then:

i) If $\left\|u_{0}\right\|_{\dot{H}^{1}}<\|W\|_{\dot{H}^{1}}$, the solution exists for all time and scatters.

ii) If $\left\|u_{0}\right\|_{L^{2}}<\infty,\left\|u_{0}\right\|_{\dot{H}^{1}}>\|W\|_{\dot{H}^{1}}$, then $T_{+}\left(u_{0}\right)<+\infty, T_{-}\left(u_{0}\right)<+\infty$.

Remark 1. Recently, Killip-Vişan [29] have combined the ideas of the proof of Theorem 2, as applied to NLS in [10], with another important new idea, to extend Theorem 1 to the non-radial case for $N \geq 5$.

The case where the radial assumption is not needed in dimensions $3 \leq N \leq 6$ is the one of (2). We have:

Theorem 2 (Kenig-Merle [23]). For the focusing energy critical (NLW), where $3 \leq N \leq 6$, consider $\left(u_{0}, u_{1}\right) \in \dot{H}^{1} \times L^{2}$ such that $E\left(\left(u_{0}, u_{1}\right)\right)<E((W, 0))$. Then:

i) If $\left\|u_{0}\right\|_{\dot{H}^{1}}<\|W\|_{\dot{H}^{1}}$, the solution exists for all time and scatters.

ii) If $\left\|u_{0}\right\|_{\dot{H}^{1}}>\|W\|_{\dot{H}^{1}}$, then $T_{ \pm}\left(u_{0}\right)<+\infty$.

I will sketch the proofs of these two theorems and the outline of the general method in these lectures. The method has found other interesting applications:

\section{Mass Critical NLS:}

$$
\begin{cases}i \partial_{t} u+\Delta u \pm|u|^{4 / N} u=0, & (x, t) \in \mathbb{R}^{N} \times \mathbb{R}, \\ \left.u\right|_{t=0}=u_{0}, & N \geq 3 .\end{cases}
$$

Here, $\left\|u_{0}\right\|_{L^{2}}$ is the critical norm. The analog of Theorem 1 was obtained, for $u_{0}$ radial, by Tao-Vişan-Zhang [50], Killip-Tao-Vişan [28], Killip-Vişan-Zhang [30], using our proof scheme for $N \geq 2$. (In the focusing case one needs to assume $\left\|u_{0}\right\|_{L^{2}}<\|Q\|_{L^{2}}$, where $Q$ is the ground state, i.e., the non-negative solution of the elliptic equation $\triangle Q+Q^{1+4 / N}=Q$.) The case $N=1$ is open. 
Corotational wave maps into $S^{2}, 4 \mathrm{D}$ Yang-Mills in the radial case: Consider the wave map system

$$
\square u=A(u)(D u, D u) \perp T_{u} M
$$

where $u=\left(u^{1}, \ldots, u^{d}\right): \mathbb{R} \times \mathbb{R}^{N} \rightarrow M \hookrightarrow \mathbb{R}^{d}$, where the target manifold $M$ is isometrically embedded in $\mathbb{R}^{d}$, and $A(u)$ is the second fundamental form for $M$ at $u$. We consider the case $M=S^{2} \subset \mathbb{R}^{3}$. The critical space here is $\left(u_{0}, u_{1}\right) \in$ $\dot{H}^{N / 2} \times \dot{H}^{N-2 / 2}$, so that when $N=2$, the critical space is $\dot{H}^{1} \times L^{2}$. It is known that for small data in $\dot{H}^{1} \times L^{2}$ we have global existence and scattering (Tătaru [52], [53], Tao [47]). Moreover, Rodnianski-Sterbenz [39] and Krieger-Schlag-Tătaru [31] showed that there can be finite time blow-up for large data. In earlier work, Struwe [45] had considered the case of co-rotational maps. These are maps which have a special form. Writing the metric on $S^{2}$ in the form $(\rho, \theta), \rho>0, \theta \in S^{1}$, with $d s^{2}=d \rho^{2}+g(\rho)^{2} d \theta^{2}$, where $g(\rho)=\sin \rho$, we consider, using $(r, \phi)$ as polar coordinates in $\mathbb{R}^{2}$, maps of the form $\rho=v(r, t), \theta=\phi$. These are the co-rotational maps and Krieger-Schlag-Tătaru [31] exhibited blow-up for corotational maps. There is a stationary solution $Q$, which is a non-constant harmonic map of least energy. Struwe proved that if $E(v) \leq E(Q)$, then $v$ and the corresponding wave map $u$ are global in time. Using our method, in joint work of Cote-Kenig-Merle [9] we show that, in addition, there is an alternative: $v \equiv Q$ or the solution scatters. We also prove the corresponding results for radial solutions of the Yang-Mills equations in the critical energy space in $\mathbb{R}^{4}$ (see [9]).

Cubic NLS in 3D: Consider the classic cubic NLS in 3D:

$$
\left\{\begin{array}{l}
i \partial_{t} u+\triangle u \mp|u|^{2} u=0, \quad(x, t) \in \mathbb{R}^{3} \times \mathbb{R}, \\
\left.u\right|_{t=0}=u_{0} \in \dot{H}^{1 / 2}\left(\mathbb{R}^{3}\right) .
\end{array}\right.
$$

Here $\dot{H}^{1 / 2}$ is the critical space, "-" corresponds to defocusing and "+" to focusing. In the focusing case, Duyckaerts-Holmer-Roudenko [10] adapted our method to show that if $u_{0} \in \dot{H}^{1}\left(\mathbb{R}^{3}\right)$ and $M\left(u_{0}\right) E\left(u_{0}\right)<M(Q) E(Q)$, where

$$
M\left(u_{0}\right)=\int\left|u_{0}\right|^{2}, \quad E\left(u_{0}\right)=\frac{1}{2} \int\left|\nabla u_{0}\right|^{2}-\frac{1}{4} \int\left|u_{0}\right|^{4},
$$

and $Q$ is the ground state, i.e., the positive solution to the elliptic equation

$$
-Q+\triangle Q+|Q|^{2} Q=0
$$

then if $\left\|u_{0}\right\|_{L^{2}}\left\|\nabla u_{0}\right\|_{L^{2}}>\|Q\|_{L^{2}}\|\nabla Q\|_{L^{2}}$, we have "blow-up" in finite time, while if $\left\|u_{0}\right\|_{L^{2}}\left\|\nabla u_{0}\right\|_{L^{2}}<\|Q\|_{L^{2}}\|\nabla Q\|_{L^{2}}$, then $u$ exists for all time and scatters. In joint work with Merle [24] we have considered the defocusing case. We have shown, using this circle of ideas, that if $\sup _{0<t<T_{+}\left(u_{0}\right)}\|u(t)\|_{\dot{H}^{1 / 2}}<\infty$, then $T_{+}\left(u_{0}\right)=+\infty$ and $u$ scatters. We would like to point out that the fact that $T_{+}\left(u_{0}\right)=+\infty$ is analogous to the $L^{3, \infty}$ result of Escauriaza-Seregin-Sverak for Navier-Stokes [11]. 
We now turn to the proofs of Theorems 1 and 2. We start with Theorem 1. We are thus considering

$$
\left\{\begin{array}{l}
i \partial_{t} u+\triangle u+|u|^{4 / N-2} u=0, \quad(x, t) \in \mathbb{R}^{N} \times \mathbb{R} \\
\left.u\right|_{t=0}=u_{0} \in \dot{H}^{1}
\end{array}\right.
$$

Let us start with a quick review of the "local Cauchy problem" theory. Besides the norm $\|f\|_{S(I)}=\|f\|_{L_{I}^{2(N+2) / N-2} L_{x}^{2(N+2) / N-2}}$ introduced earlier, we need the norm $\|f\|_{W(I)}=\|f\|_{L_{I}^{2(N+2) / N-2} L_{x}^{2(N+2) / N^{2}+4}}$.

Theorem 3 ([7], [25]). Assume that $u_{0} \in \dot{H}^{1}\left(\mathbb{R}^{N}\right),\left\|u_{0}\right\|_{\dot{H}^{1}} \leq A$. Then, for $3 \leq$ $N \leq 6$, there exists $\delta=\delta(A)>0$ such that if $\left\|e^{i t \Delta} u_{0}\right\|_{S(I)} \leq \delta, 0 \in \stackrel{\circ}{I}$, there exists a unique solution to (4) in $\mathbb{R}^{N} \times I$, with $u \in C\left(I ; \dot{H}^{1}\right)$ and $\|\nabla u\|_{W(I)}<+\infty$, $\|u\|_{S(I)} \leq 2 \delta$. Moreover, the mapping $u_{0} \in \dot{H}^{1}\left(\mathbb{R}^{N}\right) \rightarrow u \in C\left(I ; \dot{H}^{1}\right)$ is Lipschitz.

The proof is by fixed point. The key ingredients are the following "Strichartz estimates" [43], [21]:

$$
\left\{\begin{array}{c}
\left\|\nabla e^{i t \Delta} u_{0}\right\|_{W(-\infty,+\infty)} \leq C\left\|u_{0}\right\|_{H^{1}} \\
\left\|\nabla \int_{0}^{t} e^{i\left(t-t^{\prime}\right) \Delta} g\left(\cdot, t^{\prime}\right) d t^{\prime}\right\|_{W(-\infty,+\infty)} \leq C\|g\|_{L_{t}^{2} L_{x}^{2 N / N+2}} \\
\sup _{t}\left\|\nabla \int_{0}^{t} e^{i\left(t-t^{\prime}\right) \Delta} g\left(\cdot, t^{\prime}\right) d t^{\prime}\right\|_{L^{2}} \leq C\|g\|_{L_{t}^{2} L_{x}^{2 N / N+2}}
\end{array}\right.
$$

and the following Sobolev embedding

$$
\|v\|_{S(I)} \leq C\|\nabla v\|_{W(I)},
$$

and the observation that $\left|\nabla\left(|u|^{4 / N-2} u\right)\right| \leq C|\nabla u||u|^{4 / N-2}$, so that

$$
\left\|\nabla\left(|u|^{4 / N-2} u\right)\right\|_{L_{I}^{2} L_{x}^{2 N / N+2}} \lesssim\|u\|_{S(I)}^{4 / N-2}\|\nabla u\|_{W(I)} .
$$

Remark 2. Because of (5), (6), there exists $\tilde{\delta}$ such that if $\left\|u_{0}\right\|_{\dot{H}^{1}} \leq \tilde{\delta}$, the hypothesis of the Theorem is verified for $I=(-\infty,+\infty)$. Moreover, given $u_{0} \in \dot{H}^{1}$, we can find $I$ such that $\left\|e^{i t \Delta} u_{0}\right\|_{S(I)}<\delta$, so that the Theorem applies. It is then easy to see that given $u_{0} \in \dot{H}^{1}$, there exists a maximal interval $I=\left(-T_{-}\left(u_{0}\right), T_{+}\left(u_{0}\right)\right)$ where $u \in C\left(I^{\prime} ; \dot{H}^{1}\right) \cap\left\{\nabla u \in W\left(I^{\prime}\right)\right\}$ of all $I^{\prime} \subset \subset I$ is defined. We call $I$ the maximal interval of existence. It is easy to see that for all $t \in I$, we have

$$
E(u(t))=\frac{1}{2} \int|\nabla u(t)|^{2}-\frac{1}{2^{*}} \int|u|^{2^{*}}=E\left(u_{0}\right) .
$$

We also have the "standard finite time blow-up criterion": if $T_{+}\left(u_{0}\right)<\infty$, then $\|u\|_{S\left(\left[0, T_{+}\left(u_{0}\right)\right)\right.}=+\infty$. 
We next turn to another fundamental result in the "local Cauchy theory", the so called "Perturbation Theorem".

Perturbation Theorem 4 (see [49], [25], [22]). Let $I=[0, L), L \leq+\infty$, and $\tilde{u}$ defined on $\mathbb{R}^{N} \times I$ be such that

$$
\sup _{t \in I}\|\tilde{u}\|_{\dot{H}^{1}} \leq A, \quad\|\tilde{u}\|_{S(I)} \leq M, \quad\|\nabla \tilde{u}\|_{W(I)}<+\infty
$$

and verify (in the sense of the integral equation)

$$
i \partial_{t} \tilde{u}+\triangle \tilde{u}+|\tilde{u}|^{4 / N-2} \tilde{u}=e \quad \text { on } \mathbb{R}^{N} \times I,
$$

and let $u_{0} \in \dot{H}^{1}$ be such that $\left\|u_{0}-\tilde{u}(0)\right\|_{\dot{H}^{1}} \leq A^{\prime}$. Then there exists $\epsilon_{0}=\epsilon_{0}(M, A$, $\left.A^{\prime}\right)$ such that, if $0 \leq \epsilon \leq \epsilon_{0}$ and $\|\nabla e\|_{L_{I}^{2} L_{x}^{2 N / N+2}} \leq \epsilon,\left\|e^{i t \Delta}\left[u_{0}-\tilde{u}(0)\right]\right\|_{S(I)} \leq \epsilon$, then there exists a unique solution $u$ to (4) on $\mathbb{R}^{N} \times I$, such that

$$
\|u\|_{S(I)} \leq C\left(A, A^{\prime}, M\right) \quad \text { and } \quad \sup _{t \in I}\|u(t)-\tilde{u}(t)\|_{H^{1}} \leq C\left(A, A^{\prime}, M\right)\left(A^{\prime}+\epsilon\right)^{\beta},
$$

where $\beta>0$.

For the details of the proof, see [22]. This result has several important consequences:

Corollary 1. Let $K \subset \dot{H}^{1}$ be such that $\bar{K}$ is compact. Then there exist $T_{+, \bar{K}}, T_{-, \bar{K}}$ such that for all $u_{0} \in K$ we have $T_{+}\left(u_{0}\right) \geq T_{+, \bar{K}}, T_{-}\left(u_{0}\right) \geq T_{-, \bar{K}}$.

Corollary 2. Let $\tilde{u}_{0} \in \dot{H^{1}},\left\|\tilde{u}_{0}\right\|_{\dot{H}^{1}} \leq A$, and let $\tilde{u}$ be the solution of (4), with maximal interval $\left(-T_{-}\left(\tilde{u}_{0}\right), T_{+}\left(\tilde{u}_{0}\right)\right)$. Assume that $u_{0, n} \rightarrow \tilde{u}_{0}$ in $\dot{H}^{1}$, with corresponding solution $u_{n}$. Then $T_{+}\left(\tilde{u}_{0}\right) \leq \underline{\lim } T_{+}\left(u_{0, n}\right), T_{-}\left(\tilde{u}_{0}\right) \leq \underline{\lim } T_{-}\left(u_{0, n}\right)$ and for $t \in\left(-T_{-}\left(\tilde{u}_{0}\right), T_{+}\left(\tilde{u}_{0}\right)\right), u_{n}(t) \rightarrow \tilde{u}(t)$ in $\dot{H}^{1}$.

Before we start with our sketch of the proof of Theorem 1, we will review the classic argument of Glassey [15] for blow-up in finite time. Thus, assume $u_{0} \in \dot{H}^{1}$, $\int|x|^{2}\left|u_{0}(x)\right|^{2} d x<\infty$ and $E\left(u_{0}\right)<0$. Let $I$ be the maximal interval of existence. One easily shows that, for $t \in I, y(t)=\int|x|^{2}|u(x, t)|^{2} d x<+\infty$. In fact,

$$
y^{\prime}(t)=4 \operatorname{Im} \int \bar{u} \nabla u \cdot x, \quad \text { and } \quad y^{\prime \prime}(t)=8\left[\int|\nabla u(x, t)|^{2}-\int|u(x, t)|^{2^{*}}\right] .
$$

Hence, if $E\left(u_{0}\right)<0, E(u(t))=E\left(u_{0}\right)<0$, so that

$$
\frac{1}{2} \int|\nabla u(t)|^{2}-|u(t)|^{2^{*}}=E\left(u_{0}\right)+\left(\frac{1}{2^{*}}-\frac{1}{2}\right) \int|u(t)|^{2^{*}} \leq E\left(u_{0}\right)<0,
$$

and $y^{\prime \prime}(t)<0$. But then, if $I$ is infinite, since $y(t)>0$ we obtain a contradiction. We now start with our sketch of the proof of Theorem 1. 
Step 1: Variational estimates. (These are not needed in defocusing problems.) Recall that $W(x)=\left(1+|x|^{2} / N(N-2)\right)^{-(N-2) / 2}$ is a stationary solution of (4). It solves the elliptic equation $\triangle W+|W|^{4 / N-2} W=0, W \geq 0, W$ is radially decreasing, $W \in \dot{H}^{1}$. By the invariances of the equation,

$$
W_{\theta_{0}, x_{0}, \lambda_{0}}(x)=e^{i \theta_{0}} \lambda_{0}^{N-2 / 2} W\left(\lambda_{0}\left(x-x_{0}\right)\right)
$$

is still a solution. Aubin and Talenti [1], [46] gave the following variational characterization of $W$ : let $C_{N}$ be the best constant in the Sobolev embedding $\|u\|_{L^{2^{*}}} \leq$ $C_{N}|| \nabla u \|_{L^{2}}$. Then $\|u\|_{L^{2^{*}}}=C_{N}\|\nabla u\|_{L^{2}}, u \not \equiv 0$, if and only if $u=W_{\theta_{0}, x_{0}, \lambda_{0}}$ for some $\left(\theta_{0}, x_{0}, \lambda_{0}\right)$. Note that by the elliptic equation, $\int|\nabla W|^{2}=\int|W|^{2^{*}}$. Also, $C_{N}\|\nabla W\|=\|W\|_{L^{2 *}}$, so that

$$
C_{N}^{2}\|\nabla W\|^{2}=\left(\int|\nabla W|^{2}\right)^{\frac{N-2}{N}} .
$$

Hence, $\int|\nabla W|^{2}=1 / C_{N}^{N}$, and

$$
E(W)=\left(\frac{1}{2}-\frac{1}{2^{*}}\right) \int|\nabla W|^{2}=\frac{1}{N C_{N}^{N}}
$$

Lemma 1. Assume that $\|\nabla v\|<\|\nabla W\|$ and that $E(v) \leq\left(1-\delta_{0}\right) E(W), \delta_{0}>0$. Then there exists $\bar{\delta}=\bar{\delta}\left(\delta_{0}\right)$ so that:

i) $\|\nabla v\|^{2} \leq(1-\bar{\delta})\|\nabla W\|^{2}$.

ii) $\int|\nabla v|^{2}-|v|^{2^{*}} \geq \bar{\delta}|| \nabla v \|^{2}$.

iii) $E(v) \geq 0$.

Proof. Let

$$
f(y)=\frac{1}{2} y-\frac{C_{N}^{2^{*}}}{2^{*}} y^{2^{*} / 2}, \quad \bar{y}=\|\nabla v\|^{2} .
$$

Note that $f(0)=0, f(y)>0$ for $y$ near $0, y>0$, and that

$$
f^{\prime}(y)=\frac{1}{2}-\frac{C_{N}^{2^{*}}}{2^{*}} y^{2^{*} / 2-1}
$$

so that $f^{\prime}(y)=0$ if and only if $y=y_{c}=\frac{1}{C_{N}}=\|\nabla W\|^{2}$. Also, $f\left(y_{c}\right)=\frac{1}{N C_{N}}=$ $E(W)$. Since $0 \leq \bar{y}<y_{c}, f(\bar{y}) \leq\left(1-\delta_{0}\right) f\left(y_{c}\right), f$ is non-negative and strictly increasing between 0 and $y_{c}$, and $f^{\prime \prime}\left(y_{c}\right) \neq 0$, we have $0 \leq f(\bar{y}), \bar{y} \leq(1-\bar{\delta}) y_{c}=$ $(1-\bar{\delta})\|\nabla W\|^{2}$. This shows i). 
For ii), note that

$$
\begin{aligned}
\int|\nabla v|^{2}-|v|^{2^{*}} & \geq \int|\nabla v|^{2}-C_{N}^{2^{*}}\left(\int|\nabla v|^{2}\right)^{2^{*} / 2} \\
& =\int|\nabla v|^{2}\left[1-C_{N}^{2^{*}}\left(\int|\nabla v|^{2}\right)^{2 / N-2}\right] \\
& \geq \int|\nabla v|^{2}\left[1-C_{N}^{2^{*}}(1-\bar{\delta})^{2 / N-2}\left(\int|\nabla W|^{2}\right)^{2 / N-2}\right] \\
& =\int|\nabla v|^{2}\left[1-(1-\bar{\delta})^{2 / N-2}\right],
\end{aligned}
$$

which gives ii).

Note from this that if $\left\|\nabla u_{0}\right\|<\|\nabla W\|$, then $E\left(u_{0}\right) \geq 0$, i.e., iii) holds.

This static lemma immediately has dynamic consequences.

Corollary 3 (Energy Trapping). Let $u$ be a solution of (4) with maximal interval $I$, $\left\|\nabla u_{0}\right\|<\|\nabla W\|, E\left(u_{0}\right)<E(W)$. Choose $\delta_{0}>0$ such that $E\left(u_{0}\right) \leq\left(1-\delta_{0}\right) E(W)$. Then, for each $t \in I$, we have:

i) $\|\nabla u(t)\|^{2} \leq(1-\bar{\delta})\|\nabla W\|, \quad E(u(t)) \geq 0$.

ii) $\int|\nabla u(t)|^{2}-|u(t)|^{2^{*}} \geq \bar{\delta} \int|\nabla u(t)|^{2}$ ("coercivity").

iii) $E(u(t)) \approx\|\nabla u(t)\|^{2} \approx\left\|\nabla u_{0}\right\|^{2}$, with comparability constants which depend on $\delta_{0}$ ("uniform bound").

Proof. The statements follow from continuity of the flow, conservation of energy and the previous Lemma.

Note that iii) gives uniform bounds on $\|\nabla u(t)\|$. However, this is a long way from giving Theorem 1 .

Remark 3. Let $u_{0} \in \dot{H}^{1}, E\left(u_{0}\right)<E(W)$, but $\left\|\nabla u_{0}\right\|^{2}>\|\nabla W\|^{2}$. If we choose $\delta_{0}$ so that $E\left(u_{0}\right) \leq\left(1-\delta_{0}\right) E(W)$, we can conclude, as in the proof of the Lemma, that $\int|\nabla u(t)|^{2} \geq(1+\bar{\delta}) \int|\nabla W|^{2}, t \in I$. But then,

$$
\begin{aligned}
\int|\nabla u(t)|^{2}-|u(t)|^{2^{*}} & =2^{*} E\left(u_{0}\right)-\frac{2}{N-2} \int|\nabla u|^{2} \\
& \leq 2^{*} E(W)-\frac{2}{N-2} \frac{1}{C_{N}^{N}}-\frac{2 \bar{\delta}}{N-2} \frac{1}{C_{N}^{N}} \\
& =-\frac{2 \bar{\delta}}{(N-2) C_{N}^{N}}<0 .
\end{aligned}
$$

Hence, if $\int|x|^{2}\left|u_{0}(x)\right|^{2} d x<\infty$, Glassey's proof shows that $I$ cannot be infinite. If $u_{0}$ is radial, $u_{0} \in L^{2}$, using a "local virial identity" (which we will see momentarily) one can see that the same result holds. 
Step 2: Concentration-compactness procedure. We now turn to the proof of i) in Theorem 1. By our variational estimates, if $E\left(u_{0}\right)<E(W),\left\|\nabla u_{0}\right\|^{2}<\|\nabla W\|^{2}$, if $\delta_{0}$ is chosen so that $E\left(u_{0}\right) \leq\left(1-\delta_{0}\right) E(W)$, recall that

$$
E(u(t)) \approx\|\nabla u(t)\|^{2} \approx\left\|\nabla u_{0}\right\|^{2}
$$

$t \in I$, with constants depending only on $\delta_{0}$. Recall also that if $\left\|\nabla u_{0}\right\|^{2}<\|\nabla W\|^{2}$, $E\left(u_{0}\right) \geq 0$. It now follows from the "local Cauchy theory" that if $\left\|\nabla u_{0}\right\|^{2}<$ $\|\nabla W\|^{2}$ and $E\left(u_{0}\right) \leq \eta_{0}, \eta_{0}$ small, then $I=(-\infty,+\infty)$ and $\|u\|_{S(-\infty,+\infty)}<\infty$, so that $u$ scatters. Consider now

$$
\begin{aligned}
G=\{E: 0 & <E<E(W): \\
& \text { if } \left.\left\|\nabla u_{0}\right\|^{2}<\|\nabla W\|^{2} \text { and } E\left(u_{0}\right)<E \text {, then }\|u\|_{S(I)}<\infty\right\}
\end{aligned}
$$

and $E_{c}=\sup G$. Then $0<\eta_{0} \leq E_{c} \leq E(W)$ and if $\left\|\nabla u_{0}\right\|^{2}<\|\nabla W\|^{2}, E\left(u_{0}\right)<$ $E_{c}, I=(-\infty,+\infty), u$ scatters and $E_{c}$ is optimal with this property. Theorem $\left.1 \mathrm{i}\right)$ is the statement $E_{c}=E(W)$. We now assume $E_{c}<E(W)$ and will reach a contradiction. We now develop the concentration-compactness argument:

Proposition 1. There exists $u_{0, c} \in \dot{H}^{1},\left\|\nabla u_{0, c}\right\|^{2}<\|\nabla W\|^{2}$, with $E\left(u_{0, c}\right)=E_{c}$, such that, for the corresponding solution $u_{c}$, we have $\left\|u_{c}\right\|_{S(I)}=+\infty$.

Proposition 2. For any $u_{c}$ as in Proposition 1 , with (say) $\left\|u_{c}\right\|_{S\left(I_{+}\right)}=+\infty, I_{+}=$ $I \cap[0,+\infty)$, there exist $x(t), t \in I_{+}, \lambda(t) \in \mathbb{R}^{+}, t \in I_{+}$, such that

$$
K=\left\{v(x, t)=\frac{1}{\lambda(t)^{N-2 / 2}} u\left(\frac{x-x(t)}{\lambda(t)}, t\right), t \in I_{+}\right\}
$$

has compact closure in $\dot{H}^{1}$.

The proof of Propositions 1 and 2 follows a "general procedure" which uses a "profile decomposition", the variational estimates and the "Perturbation Theorem". The idea of the decomposition is somehow a time-dependent version of the concentration-compactness method of P. L. Lions, when the "local Cauchy theory" is done in the critical space. It was introduced independently by Bahouri-Gérard [2] for the wave equation and by Merle-Vega for the $L^{2}$ critical NLS [35]. The version needed for Theorem 1 is due to Keraani [27]. This is the evolution analog of the elliptic "bubble decomposition", which goes back to work of Brézis-Coron [5].

Theorem 5 (Keraani [27]). Let $\left\{v_{0, n}\right\} \subset \dot{H}^{1}$, with $\left\|v_{0, n}\right\|_{\dot{H}^{1}} \leq$ A. Assume that $\left\|e^{i t \Delta} v_{0, n}\right\|_{S(-\infty,+\infty)} \geq \delta>0$. Then there exists a subsequence of $\left\{v_{0, n}\right\}$ and $a$ sequence $\left\{V_{0, j}\right\}_{j=1}^{\infty} \subset \dot{H}^{1}$ and triples $\left\{\left(\lambda_{j, n}, x_{j, n}, t_{j, n}\right)\right\} \subset \mathbb{R}^{+} \times \mathbb{R}^{N} \times \mathbb{R}$, with

$$
\frac{\lambda_{j, n}}{\lambda_{j^{\prime}, n}}+\frac{\lambda_{j^{\prime}, n}}{\lambda_{j, n}}+\frac{\left|t_{j, n}-t_{j^{\prime}, n}\right|}{\lambda_{j, n}^{2}}+\frac{\left|x_{j, n}-x_{j^{\prime}, n}\right|}{\lambda_{j, n}} \underset{n \rightarrow \infty}{\longrightarrow} \infty,
$$

for $j \neq j^{\prime}$ (we say that $\left\{\left(\lambda_{j, n}, x_{j, n}, t_{j, n}\right)\right\}$ is orthogonal), such that 
i) $\left\|V_{0,1}\right\|_{H^{1}} \geq \alpha_{0}(A)>0$.

ii) If $V_{j}^{l}(x, t)=e^{i t \Delta} V_{0, j}$, then we have, for each $J$,

$$
v_{0, n}=\sum_{j=1}^{J} \frac{1}{\lambda_{j, n}^{N-2 / 2}} V_{j}^{l}\left(\frac{x-x_{j, n}}{\lambda_{j, n}},-\frac{t_{j, n}}{\lambda_{j, n}^{2}}\right)+w_{n}^{J},
$$

where $\frac{\lim }{n}\left\|e^{i t \Delta} w_{n}^{J}\right\|_{S(-\infty,+\infty)} \underset{J \rightarrow \infty}{\longrightarrow} 0$, and for each $J \geq 1$ we have

iii) $\left\|\nabla v_{0, n}\right\|^{2}=\sum_{j=1}^{J}\left\|\nabla V_{0, j}\right\|^{2}+\left\|\nabla w_{n}^{J}\right\|^{2}+o(1)$ as $n \rightarrow \infty$ and

$$
E\left(v_{0, n}\right)=\sum_{j=1}^{J} E\left(V_{j}^{l}\left(-\frac{t_{j, n}}{\lambda_{j, n}^{2}}\right)\right)+E\left(w_{n}^{J}\right)+o(1) \text { as } n \rightarrow \infty .
$$

Further general remarks:

Remark 4. Because of the continuity of $u(t), t \in I$, in $\dot{H}^{1}$, in Proposition 2 we can construct $\lambda(t), x(t)$ continuous in $\left[0, T_{+}\left(u_{0}\right)\right)$, with $\lambda(t)>0$.

Remark 5. Because of scaling and the compactness of $\bar{K}$ above, if $T_{+}\left(u_{0, c}\right)<\infty$, one always has that $\lambda(t) \geq C_{0}(K) /\left(T_{+}\left(u_{0}, c\right)-t\right)^{\frac{1}{2}}$.

Remark 6. If $T_{+}\left(u_{0, c}\right)=+\infty$, we can always find another (possibly different) critical element $v_{c}$ with a corresponding $\tilde{\lambda}$ so that $\tilde{\lambda}(t) \geq A_{0}>0$ for $t \in\left[0, T_{+}\left(v_{0, c}\right)\right)$. (Again by compactness of $\bar{K}$.)

Remark 7. One can use the "profile decomposition" to also show that there exists a decreasing function $g:\left(0, E_{c}\right] \rightarrow[0,+\infty)$ so that if $\left\|\nabla u_{0}\right\|^{2}<\|\nabla W\|^{2}$ and $E\left(u_{0}\right) \leq E_{c}-\eta$, then $\|u\|_{S(-\infty,+\infty)} \leq g(\eta)$.

Remark 8. In the "profile decomposition", if all the $v_{0, n}$ are radial, the $V_{0, j}$ can be chosen radial and $x_{j, n} \equiv 0$. We can repeat our procedure restricted to radial data and conclude the analog of Propositions 1 and 2 with $x(t) \equiv 0$.

The final step in the proof is then:

\section{Step 3: Rigidity Theorem.}

Theorem 6 (Rigidity). Let $u_{0} \in \dot{H}^{1}, E\left(u_{0}\right)<E(W),\left\|\nabla u_{0}\right\|^{2}<\|\nabla W\|^{2}$. Let $u$ be the solution of (4), with maximal interval $I=\left(-T_{-}\left(u_{0}\right), T_{+}\left(u_{0}\right)\right)$. Assume that there exists $\lambda(t)>0$, defined for $t \in\left[0, T_{+}\left(u_{0}\right)\right)$, such that

$$
K=\left\{v(x, t)=\frac{1}{\lambda(t)^{N-2 / 2}} u\left(\frac{x}{\lambda(t)}, t\right), t \in\left[0, T_{+}\left(u_{0}\right)\right)\right\}
$$

has compact closure in $\dot{H}^{1}$. Assume also that, if $T_{+}\left(u_{0}\right)<\infty$,

$$
\lambda(t) \geq C_{0}(K) /\left(T_{+}\left(u_{0}, c\right)-t\right)^{\frac{1}{2}} \text { and }
$$

if $T_{+}\left(u_{0}\right)=\infty$, that $\lambda(t) \geq A_{0}>0$ for $t \in[0,+\infty)$. Then $T_{+}\left(u_{0}\right)=+\infty, u_{0} \equiv 0$. 
To prove this, we split two cases:

Case 1: $T_{+}\left(u_{0}\right)<+\infty$. (So that $\lambda(t) \rightarrow+\infty$ as $t \rightarrow T_{+}\left(u_{0}\right)$.)

Fix $\phi$ radial, $\phi \in C_{0}^{\infty}, \phi \equiv 1$ on $|x| \leq 1, \operatorname{supp} \phi \subset\{|x|<2\}$. Set $\phi_{R}(x)=$ $\phi(x / R)$ and define

$$
y_{R}(t)=\int|u(x, t)|^{2} \phi_{R}(x) d x .
$$

Then $y_{r}^{\prime}(t)=2 \operatorname{Im} \int \bar{u} \nabla u \nabla \phi_{R}$, so that

$$
\left|y_{R}^{\prime}(t)\right| \leq C\left(\int|\nabla u|^{2}\right)^{1 / 2}\left(\int \frac{|u|^{2}}{|x|^{2}}\right)^{1 / 2} \leq C|| \nabla W \|^{2},
$$

by Hardy's inequality and our variational estimates. Note that $C$ is independent of $R$. Next, we note that, for each $R>0$,

$$
\lim _{t \uparrow T_{+}\left(u_{0}\right)} \int_{|x|<R}|u(x, t)|^{2} d x=0 .
$$

In fact, $u(x, t)=\lambda(t)^{N-2 / 2} v(\lambda(t) x, t)$, so that

$$
\begin{aligned}
\int_{|x|<R}|u(x, t)|^{2} d x & =\lambda(t)^{-2} \int_{|y|<R \lambda(t)}|v(y, t)|^{2} d y \\
& =\lambda(t)^{-2} \int_{|y|<\epsilon R \lambda(t)}|v(y, t)|^{2} d y \\
& +\lambda(t)^{-2} \int_{\epsilon R \lambda(t) \leq|y|<R \lambda(t)}|v(y, t)|^{2} d y \\
& =A+B . \\
A \leq \lambda(t)^{-2}(\epsilon R \lambda(t))^{2}\|v\|_{L^{2^{*}}}^{2} \leq C \epsilon^{2} R^{2}\|\nabla W\|^{2} &
\end{aligned}
$$

which is small with $\epsilon$.

$$
B \leq \lambda(t)^{-2}(R \lambda(t))^{2}\|v\|_{L^{2^{*}}(|y| \geq \epsilon R \lambda(t))}^{2} \underset{t \rightarrow T_{+}\left(u_{0}\right)}{\longrightarrow} 0,
$$

(since $\lambda(t) \uparrow+\infty$ as $\left.t \rightarrow T_{+}\left(u_{0}\right)\right)$ using the compactness of $\bar{K}$. But then $y_{R}(0) \leq$ $C T_{+}\left(u_{0}\right)\|\nabla W\|^{2}$, by the fundamental theorem of calculus. Thus, letting $R \rightarrow \infty$, we see that $u_{0} \in L^{2}$, but then, using the conservation of the $L^{2}$ norm, we see that $\left\|u_{0}\right\|_{L^{2}}=\left\|u\left(T_{+}\left(u_{0}\right)\right)\right\|_{L^{2}}=0$, so that $u_{0} \equiv 0$.

Case 2: $T_{+}\left(u_{0}\right)=+\infty$. First note that the compactness of $\bar{K}$, together with $\lambda(t) \geq A_{0}>0$, gives that, given $\epsilon>0$, there exists $R(\epsilon)>0$ such that, for all $t \in[0,+\infty)$,

$$
\int_{|x|>R(\epsilon)}|\nabla u|^{2}+|u|^{2^{*}}+\frac{|u|^{2}}{|x|^{2}} \leq \epsilon .
$$


Pick $\delta_{0}>0$ so that $E\left(u_{0}\right) \leq\left(1-\delta_{0}\right) E(W)$. Recall that, by our variational estimates, we have that $\int|\nabla u(t)|^{2}-|u(t)|^{2^{*}} \geq C_{\delta_{0}}|| \nabla u_{0} \|_{L^{2}}^{2}$. If $\left\|\nabla u_{0}\right\|_{L^{2}} \neq 0$, using the smallness of tails, we see that, for $R>R_{0}$,

$$
\int_{|x|<R}|\nabla u(t)|^{2}-|u(t)|^{2^{*}} \geq C_{\delta_{0}}\left\|\nabla u_{0}\right\|_{L^{2}}^{2} .
$$

Choose now $\psi \in C_{0}^{\infty}$ radial with $\psi(x)=|x|^{2}$ for $|x| \leq 1$, supp $\psi \subset\{|x| \leq 2\}$. Define

$$
z_{R}(t)=\int|u(x, t)|^{2} R^{2} \psi(x / R) d x .
$$

Similar computations to Glassey's blow-up proof give:

$$
z_{R}^{\prime}(t)=2 R \operatorname{Im} \int \bar{u} \nabla u \nabla \psi(x / R)
$$

and

$$
\begin{aligned}
z_{R}^{\prime \prime}(t) & =4 \sum_{l, j} \operatorname{Re} \int \partial_{x_{l}} \partial_{x_{j}} \psi(x / R) \partial_{x_{l}} u \partial_{x_{j}} \bar{u} \\
& -\frac{1}{R^{2}} \int \triangle^{2} \psi(x / R)|u|^{2}-\frac{4}{N} \int \triangle \psi(x / R)|u|^{2^{*}} .
\end{aligned}
$$

Note that $\left|z_{R}^{\prime}(t)\right| \leq C_{\delta_{0}} R^{2}\left\|\nabla u_{0}\right\|^{2}$, by Cauchy-Schwartz, Hardy's inequality and our variational estimates. On the other hand,

$$
\begin{aligned}
z_{R}^{\prime \prime}(t) & \geq\left[\int_{|x| \leq R}|\nabla u(t)|^{2}-|u(t)|^{2^{*}}\right] \\
& -C\left(\int_{R \leq|x| \leq 2 R}|\nabla u(t)|^{2}+\frac{|u|^{2}}{|x|^{2}}+|u(t)|^{2^{*}}\right) \\
& \geq C|| \nabla u_{0} \|^{2},
\end{aligned}
$$

for $R$ large. Integrating in $t$, we obtain $z_{R}^{\prime}(t)-z_{R}^{\prime}(0) \geq C t\left\|\nabla u_{0}\right\|^{2}$, but

$$
\left|z_{R}^{\prime}(t)-z_{R}^{\prime}(0)\right| \leq 2 C R^{2}\left\|\nabla u_{0}\right\|^{2},
$$

which is a contradiction for $t$ large, proving Theorem $1 \mathrm{i}$ ).

Remark 9. In the defocusing case, the proof is easier since the variational estimates are not needed.

Remark 10. It is quite likely that for $N=3$, examples similar to those by P. Raphäel [38] can be constructed, of radial data $u_{0}$ for which $T_{+}\left(u_{0}\right)<\infty$ and $u$ blows up exactly on a sphere. 
We now turn to Theorem 2. We thus consider

$$
\left\{\begin{array}{l}
\partial_{t}^{2} u-\triangle u=|u|^{4 / N-2} u, \quad(x, t) \in \mathbb{R}^{N} \times \mathbb{R}, \\
\left.u\right|_{t=0}=u_{0} \in \dot{H}^{1}\left(\mathbb{R}^{n}\right), \\
\left.\partial_{t} u\right|_{t=0}=u_{1} \in L^{2}\left(\mathbb{R}^{n}\right), \quad N \geq 3 .
\end{array}\right.
$$

Recall that $W(x)=\left(1+|x|^{2} / N(N-2)\right)^{-(N-2) / 2}$ is a static solution that does not scatter. The general scheme of the proof is similar to the one for Theorem 1 . We start out with a brief review of the "local Cauchy problem". We first consider the asociated linear problem,

$$
\left\{\begin{array}{l}
\partial_{t}^{2} w-\triangle w=h \\
\left.w\right|_{t=0}=w_{0} \in \dot{H}^{1}\left(\mathbb{R}^{n}\right) \\
\left.\partial_{t} w\right|_{t=0}=w_{1} \in L^{2}\left(\mathbb{R}^{N}\right) .
\end{array}\right.
$$

As is well known (see [42] for instance), the solution is given by

$$
\begin{aligned}
w(x, t) & =\cos (t \sqrt{-\triangle}) w_{0}+(-\triangle)^{-1 / 2} \sin (t \sqrt{-\triangle}) w_{1} \\
& +\int_{0}^{t}(-\triangle)^{-1 / 2} \sin ((t-s) \sqrt{-\triangle}) h(s) d s \\
& =S(t)\left(\left(w_{0}, w_{1}\right)\right)+\int_{0}^{t}(-\triangle)^{-1 / 2} \sin ((t-s) \sqrt{-\triangle}) h(s) d s .
\end{aligned}
$$

The following are the relevant Strichartz estimates: for an interval $I \subset \mathbb{R}$, let

$$
\begin{aligned}
& \|f\|_{S(I)}=\|f\|_{L_{I}^{2(N+1) / N-2} L_{x}^{2(N+1) / N-2}}, \\
& \|f\|_{W(I)}=\|f\|_{L_{I}^{2(N+1) / N-1} L_{x}^{2(N+1) / N-1}} .
\end{aligned}
$$

Then (see [14], [23])

$$
\begin{aligned}
& \sup _{t}\left\|\left(w(t), \partial_{t} w(t)\right)\right\|_{\dot{H}^{1} \times L^{2}}+\left\|D^{1 / 2} w\right\|_{W(-\infty,+\infty)}+ \\
& +\left\|\partial_{t} D^{-1 / 2} w\right\|_{W(-\infty,+\infty)}+\|w\|_{S(-\infty,+\infty)}+ \\
& +\|w\|_{L_{t}^{(N+2) / N-2} L_{x}^{2(N+2) / N-2}} \leq \\
& \leq C\left\{\left\|\left(w_{0}, w_{1}\right)\right\|_{\dot{H}^{1} \times L^{2}}+\|w\|_{L_{t}^{2(N+1) / N+3} L_{x}^{2(N+1) / N+3}}\right\} .
\end{aligned}
$$

Because of the appearance of $D^{1 / 2}$ in these estimates, we also need to use the following version of the chain rule for fractional derivatives (see [26]). 
Lemma 2. Assume $F \in C^{2}, F(0)=F^{\prime}(0)=0$, and that for all $a, b$ we have $\left|F^{\prime}(a+b)\right| \leq C\left\{\left|F^{\prime}(a)\right|+\left|F^{\prime}(b)\right|\right\}$ and $\left|F^{\prime \prime}(a+b)\right| \leq C\left\{\left|F^{\prime \prime}(a)\right|+\left|F^{\prime \prime}(b)\right|\right\}$. Then, for $0<\alpha<1, \frac{1}{p}=\frac{1}{p_{1}}+\frac{1}{p_{2}}, \frac{1}{p}=\frac{1}{r_{1}}+\frac{1}{r_{2}}+\frac{1}{r_{3}}$, we have

i) $\left\|D^{\alpha} F(u)\right\|_{L^{p}} \leq C\left\|F^{\prime}(u)\right\|_{L^{p_{1}}}\left\|D^{\alpha} u\right\|_{L^{p_{2}}}$,

ii) $\left\|D^{\alpha}(F(u)-F(v))\right\|_{L^{p}} \leq C\left[\left\|F^{\prime}(u)\right\|_{L^{p_{1}}}+\left\|F^{\prime}(v)\right\|_{L^{p_{1}}}\right]\left\|D^{\alpha}(u-v)\right\|_{L^{p_{2}}}$

$+C\left[\left\|F^{\prime \prime}(u)\right\|_{L^{r_{1}}}+\left\|F^{\prime \prime}(v)\right\|_{L^{r_{1}}}\right]\left[\left\|D^{\alpha} u\right\|_{L^{r_{2}}}+\left\|D^{\alpha} v\right\|_{L^{r_{2}}}\right]\|u-v\|_{L^{r_{3}}}$. obtain:

Using (9) and this Lemma, one can now use the same argument as for (4) to

Theorem 7 ([14], [20], [41] and [23]). Assume that

$$
\left(u_{0}, u_{1}\right) \in \dot{H}^{1} \times L^{2}, \quad\left\|\left(u_{0}, u_{1}\right)\right\|_{\dot{H}^{1} \times L^{2}} \leq A .
$$

Then, for $3 \leq N \leq 6$, there exists $\delta=\delta(A)>0$ such that if $\left\|S(t)\left(u_{0}, u_{1}\right)\right\|_{S(I)} \leq \delta$, $0 \in \stackrel{\circ}{I}$, there exists a unique solution to $(7)$ in $\mathbb{R}^{N} \times I$, with $\left(u, \partial_{t} u\right) \in C\left(I ; \dot{H}^{1} \times L^{2}\right)$ and $\left\|D^{1 / 2} u\right\|_{W(I)}+\left\|\partial_{t} D^{-1 / 2} u\right\|_{W(I)}<\infty,\|u\|_{S(I)} \leq 2 \delta$. Moreover, the mapping $\left(u_{0}, u_{1}\right) \in \dot{H}^{1} \times L^{2} \rightarrow\left(u, \partial_{t} u\right) \in C\left(I ; \dot{H}^{1} \times L^{2}\right)$ is Lipschitz.

Remark 11. Again, using (9), if $\left\|\left(u_{0}, u_{1}\right)\right\|_{\dot{H}^{1} \times L^{2}} \leq \tilde{\delta}$, the hypothesis of the Theorem is verified for $I=(-\infty,+\infty)$. Moreover, given $\left(u_{0}, u_{1}\right) \in \dot{H}^{1} \times L^{2}$, we can find $I \ni 0$ so that the hypothesis is verified on $I$. One can then define a maximal interval of existence $I=\left(-T_{-}\left(u_{0}, u_{1}\right), T_{+}\left(u_{0}, u_{1}\right)\right)$, similarly to the case of $(4)$. We also have the "standard finite time blow-up criterion": if $T_{+}\left(u_{0}, u_{1}\right)<\infty$, then $\|u\|_{S\left(0, T_{+}\left(u_{0}, u_{1}\right)\right)}=+\infty$. Also, if $T_{+}\left(u_{0}, u_{1}\right)=+\infty, u$ scatters at $+\infty$ (i.e., $\exists\left(u_{0}^{+}, u_{1}^{+}\right) \in \dot{H}^{1} \times L^{2}$ such that $\left.\left\|\left(u(t), \partial_{t} u(t)\right)-S(t)\left(u_{0}^{+}, u_{1}^{+}\right)\right\|_{\dot{H}^{1} \times L^{2}} \underset{t \uparrow+\infty}{\longrightarrow} 0\right)$ if and only if $\|u\|_{S(0,+\infty)}<+\infty$. Moreover, for $t \in I$, we have

$$
E\left(\left(u_{0}, u_{1}\right)\right)=\frac{1}{2} \int\left|\nabla u_{0}\right|^{2}+\frac{1}{2} \int u_{1}^{2}-\frac{1}{2^{*}} \int\left|u_{0}\right|^{2^{*}}=E\left(\left(u(t), \partial_{t} u(t)\right)\right) .
$$

It turns out that for (7) there is another very important conserved quantity in the energy space, namely momentum. This is crucial for us to be able to treat non-radial data. This says that, for $t \in I, \int \nabla u(t) \cdot \partial_{t} u(t)=\int \nabla u_{0} \cdot u_{1}$. Finally, the analog of the "Perturbation Theorem" also holds in this context (see [22]). All the corollaries of the Perturbation Theorem also hold.

Remark 12 (Finite speed of propagation). Recall that if $R(t)$ is the forward fundamental solution for the linear wave equation, the solution for (8) is given by (see [42])

$$
w(t)=\partial_{t} R(t) * w_{0}+R(t) * w_{1}-\int_{0}^{t} R(t-s) * h(s) d s,
$$


where $*$ stands for convolution in the $x$ variable. The finite speed of propagation is the statement that $\operatorname{supp} R(\cdot, t), \operatorname{supp} \partial_{t} R(\cdot, t) \subset \overline{B(0, t)}$. Thus, if supp $w_{0} \subset$ ${ }^{C} B\left(x_{0}, a\right), \operatorname{supp} w_{1} \subset{ }^{C} B\left(x_{0}, a\right), \operatorname{supp} h \subset{ }^{C}\left[\bigcup_{0<t<a} B\left(x_{0}, a-t\right) \times\{t\}\right]$, then $w \equiv 0$ on $\bigcup_{0<t<a} B\left(x_{0}, a-t\right) \times\{t\}$. This has important consequences for solutions of (7). If $\left(u_{0}, u_{1}\right) \equiv\left(u_{0}^{\prime}, u_{1}^{\prime}\right)$ on $B\left(x_{0}, a\right)$, then the corresponding solutions agree on $\bigcup_{0 \leq t \leq a} B\left(x_{0}, a-t\right) \times\{t\} \cap \mathbb{R}^{N} \times\left(I \cap I^{\prime}\right)$.

We now proceed with the proof of Theorem 2. As in the case of (4), the proof is broken up in three steps.

Step1: Variational estimates. Here these are immediate from the corresponding ones in (4). The summary is (we use the notation $\mathcal{E}(v)=\frac{1}{2} \int|\nabla v|^{2}-\frac{1}{2^{*}} \int|v|^{2^{*}}$ ):

Lemma 3. Let $\left(u_{0}, u_{1}\right) \in \dot{H}^{1} \times L^{2}$ be such that $E\left(\left(u_{0}, u_{1}\right)\right) \leq\left(1-\delta_{0}\right) E((W, 0))$, $\left.|| \nabla u_{0}\right|^{2}<\|\nabla W\|^{2}$. Let $u$ be the corresponding solution of $(7)$, with maximal interval I. Then there exists $\bar{\delta}=\bar{\delta}\left(\delta_{0}\right)>0$ such that, for $t \in I$, we have

i) $\|\nabla u(t)\| \leq(1-\bar{\delta})\|\nabla W\|$.

ii) $\int|\nabla u(t)|^{2}-|u(t)|^{2^{*}} \geq \bar{\delta} \int|\nabla u(t)|^{2}$.

iii) $\mathcal{E}(u(t)) \geq 0$ (and here $E\left(\left(u, \partial_{t} u\right)\right) \geq 0$ ).

iv) $E\left(\left(u, \partial_{t} u\right)\right) \approx\left\|\left(u(t), \partial_{t} u(t)\right)\right\|_{\dot{H}^{1} \times L^{2}}^{2} \approx\left\|\left(u_{0}, u_{1}\right)\right\|_{\dot{H}^{1} \times L^{2}}^{2}$, with comparability constants depending only on $\delta_{0}$.

Remark 13. If $E\left(\left(u_{0}, u_{1}\right)\right) \leq\left(1-\delta_{0}\right) E((W, 0)),\left\|\nabla u_{0}\right\|^{2}>\|\nabla W\|^{2}$, then, for $t \in I$, $\|\nabla u(t)\|^{2} \geq(1+\bar{\delta})\|\nabla W\|^{2}$. This follows from the corresponding result for (4).

We now turn to the proof of ii) in Theorem 2. We will do it for the case when $\left\|u_{0}\right\|_{L^{2}}<\infty$. For the general case, see [23]. We know that, in the situation of ii), we have

$$
\begin{gathered}
\int|\nabla u(t)|^{2} \geq(1+\bar{\delta}) \int|\nabla W|^{2}, \quad t \in I, \\
E((W, 0)) \geq E\left(\left(u(t), \partial_{t} u\right)\right)+\tilde{\delta}_{0} .
\end{gathered}
$$

Thus,

$$
\frac{1}{2^{*}} \int|u(t)|^{2^{*}} \geq \frac{1}{2} \int\left(\partial_{t} u(t)\right)^{2}+\frac{1}{2} \int|\nabla u(t)|^{2}-E((W, 0))+\tilde{\delta}_{0},
$$

so that

$$
\int|u(t)|^{2^{*}} \geq \frac{N}{N-2} \int\left(\partial_{t} u(t)\right)^{2}+\frac{N}{N-2} \int|\nabla u(t)|^{2}-2^{*} E((W, 0))+2^{*} \tilde{\delta}_{0} .
$$

Let $y(t)=\int|u(t)|^{2}$, so that $y^{\prime}(t)=2 \int u(t) \partial_{t} u(t)$. A simple calculation gives

$$
y^{\prime \prime}(t)=2 \int\left\{\left(\partial_{t} u\right)^{2}-|\nabla u(t)|^{2}+|u(t)|^{2^{*}}\right\} .
$$


Thus,

$$
\begin{aligned}
y^{\prime \prime}(t) & \geq 2 \int\left(\partial_{t} u\right)^{2}+\frac{2 N}{N-2} \int\left(\partial_{t} u\right)^{2}-2 \cdot 2^{*} E((W, 0))+ \\
& +\tilde{\tilde{\delta}}_{0}+\frac{2 N}{N-2} \int|\nabla u(t)|^{2}-2 \int|\nabla u(t)|^{2}= \\
& =\frac{4(N-1)}{N-2} \int\left(\partial_{t} u\right)^{2}+\frac{4}{N-2} \int|\nabla u(t)|^{2}- \\
& -\frac{4}{N-2} \int|\nabla W|^{2}+\tilde{\tilde{\delta}}_{0} \geq \\
& \geq \frac{4(N-1)}{N-2} \int\left(\partial_{t} u\right)^{2}+\tilde{\tilde{\delta}}_{0} .
\end{aligned}
$$

If $I \cap[0,+\infty)=[0,+\infty)$, there exists $t_{0}>0$ so that $y^{\prime}\left(t_{0}\right)>0, y^{\prime}(t)>0, t>t_{0}$. For $t>t_{0}$ we have

$$
y(t) y^{\prime \prime}(t) \geq \frac{4(N-1)}{N-2} \int\left(\partial_{t} u\right)^{2} \int u^{2} \geq\left(\frac{N-1}{N-2}\right) y^{\prime}(t)^{2},
$$

so that

or

$$
\frac{y^{\prime \prime}(t)}{y^{\prime}(t)} \geq\left(\frac{N-1}{N-2}\right) \frac{y^{\prime}(t)}{y(t)}
$$

$$
y^{\prime}(t) \geq C_{0} y(t)^{(N-1) /(N-2)}, \text { for } t>t_{0} .
$$

But, since $N-1 / N-2>1$, this leads to finite time blow-up, a contradiction.

We next turn to the proof of i) in Theorem 2 .

Step 2: Concentration-compactness procedure. Here we proceed initially in an identical manner as in the case of (4), replacing the "profile decomposition" of Keraani [27] with the corresponding one for the wave equation, due to BahouriGérard [2]. Thus, arguing by contradiction, we find a number $E_{c}$, with $0<\eta_{0} \leq$ $E_{c}<E((W, 0))$ with the property that if $E\left(\left(u_{0}, u_{1}\right)\right)<E_{c},\left\|\nabla u_{0}\right\|^{2}<\|\nabla W\|^{2}$, $\|u\|_{S(I)}<\infty$ and $E_{c}$ is optimal with this property. We will see that this leads to a contradiction. As for (4), we have:

Proposition 3. There exists

$$
\left(u_{0, c}, u_{1, c}\right) \in \dot{H}^{1} \times L^{2}, \quad\left\|\nabla u_{0, c}\right\|^{2}<\|\nabla W\|^{2}, \quad E\left(\left(u_{0, c}, u_{1, c}\right)\right)=E_{c}
$$

and such that for the corresponding solution $u_{c}$ on (7) we have $\left\|u_{c}\right\|_{S(I)}=+\infty$.

Proposition 4. For any $u_{c}$ as in Proposition 3, with (say) $\left\|u_{c}\right\|_{S\left(I_{+}\right)}=+\infty, I_{+}=$ $I \cap[0,+\infty)$, there exists $x(t) \in \mathbb{R}^{N}, \lambda(t) \in \mathbb{R}^{+}, t \in I_{+}$, such that

$$
K=\left\{v(x, t)=\left(\frac{1}{\lambda(t)^{N-2 / 2}} u_{c}\left(\frac{x-x(t)}{\lambda(t)}, t\right), \frac{1}{\lambda(t)^{N / 2}} \partial_{t} u_{c}\left(\frac{x-x(t)}{\lambda(t)}, t\right)\right): t \in I_{+}\right\}
$$

has compact closure in $\dot{H}^{1} \times L^{2}$. 
Remark 14. As in the case of (4), in Proposition 4 we can construct $\lambda(t), x(t)$ continuous in $\left[0, T_{+}\left(\left(u_{0, c}, u_{1, c}\right)\right)\right)$. Moreover, by scaling and compactness of $\bar{K}$, if $T_{+}\left(\left(u_{0, c}, u_{1, c}\right)\right)<\infty$, we have $\lambda(t) \geq C_{0}(K) /\left(T_{+}\left(\left(u_{0, c}, u_{1, c}\right)\right)-t\right)$. Also, if $T_{+}\left(\left(u_{0, c}, u_{1, c}\right)\right)=+\infty$, we can always find another (possibly different) critical element $v_{c}$, with a corresponding $\tilde{\lambda}$ so that $\tilde{\lambda}(t) \geq A>0$, for $t \in\left[0, T_{+}\left(\left(v_{0, c}, v_{1, c}\right)\right)\right)$, using the compactness of $\bar{K}$. We can also find $g:\left(0, E_{c}\right] \rightarrow[0,+\infty)$ decreasing so that if $\left\|\nabla u_{0}\right\|^{2}<\|\nabla W\|^{2}$ and $E\left(\left(u_{0, c}, u_{1, c}\right)\right) \leq E_{c}-\eta$, then $\|u\|_{S(-\infty,+\infty)} \leq g(\eta)$.

Up to here, we have used, in Step 2, only Step 1 and "general arguments". To proceed further we need to use specific features of (7) to establish further properties of critical elements.

The first one is a consequence of the finite speed of propagation and the compactness of $\bar{K}$.

Lemma 4. Let $u_{c}$ be a critical element as in Proposition 4 , with $T_{+}\left(\left(u_{0, c}, u_{1, c}\right)\right)<$ $+\infty$. (We can assume, by scaling, that $T_{+}\left(\left(u_{0, c}, u_{1, c}\right)\right)=1$.) Then there exists $\bar{x} \in \mathbb{R}^{N}$ such that $\operatorname{supp} u_{c}(\cdot, t), \operatorname{supp} \partial_{t} u_{c}(\cdot, t) \subset B(\bar{x}, 1-t), 0<t<1$.

In order to prove this Lemma, we will need the following consequence of the finite speed of propagation:

Remark 15. Let $\left(u_{0}, u_{1}\right) \in \dot{H}^{1} \times L^{2},\left\|\left(u_{0}, u_{1}\right)\right\|_{\dot{H}^{1} \times L^{2}} \leq A$. If, for some $M>0$ and $0<\epsilon<\epsilon_{0}(A)$, we have

$$
\int_{|x| \geq M}\left|\nabla u_{0}\right|^{2}+\left|u_{1}\right|^{2}+\frac{\left|u_{0}\right|^{2}}{|x|^{2}} \leq \epsilon,
$$

then for $0<t<T_{+}\left(u_{0}, u_{1}\right)$ we have

$$
\int_{|x| \geq \frac{3}{2} M+t}|\nabla u(t)|^{2}+\left|\partial_{t} u(t)\right|^{2}+|u(t)|^{2^{*}}+\frac{|u(t)|^{2}}{|x|^{2}} \leq C \epsilon
$$

Indeed, choose $\psi_{M} \in C^{\infty}, \psi_{M} \equiv 1$ for $|x| \geq \frac{3}{2} M$, with $\psi_{M} \equiv 0$ for $|x| \leq M$. Let $u_{0, M}=u_{0} \psi_{M}, u_{1, M}=u_{1} \psi_{M}$. From our assumptions, we have $\left\|\left(u_{0, M}, u_{1, M}\right)\right\|_{\dot{H}^{1} \times L^{2}} \leq C \epsilon$. If $C \epsilon_{0}<\tilde{\delta}$, where $\tilde{\delta}$ is as in the "local Cauchy theory", the corresponding solution $u_{M}$ of (7) has maximal interval $(-\infty,+\infty)$ and $\sup _{t \in(-\infty,+\infty)}\left\|\left(u_{M}(t), \partial_{t} u_{M}(t)\right)\right\|_{\dot{H}^{1} \times L^{2}} \leq 2 C \epsilon$. But, by finite speed of propagation, $u_{M} \equiv u$ for $|x| \geq \frac{3}{2} M+t, t \in\left[0, T_{+}\left(u_{0}, u_{1}\right)\right)$, which proves the Remark.

We turn to the proof of the Lemma. Recall that $\lambda(t) \geq C_{0}(K) /(1-t)$. We claim that, for any $R_{0}>0$,

$$
\lim _{t \uparrow 1} \int_{|x+x(t) / \lambda(t)| \geq R_{0}}\left|\nabla u_{c}(x, t)\right|^{2}+\left|\partial_{t} u_{c}(x, t)\right|^{2}+\frac{\left|u_{c}(x, t)\right|^{2}}{|x|^{2}}=0 .
$$

Indeed, if $\vec{v}(x, t)=\frac{1}{\lambda(t)^{N / 2}}\left(\nabla u_{c}\left(\frac{x-x(t)}{\lambda(t)}, t\right), \partial_{t} u_{c}\left(\frac{x-x(t)}{\lambda(t)}, t\right)\right)$,

$$
\int_{|x+x(t) / \lambda(t)| \geq R_{0}}\left|\nabla u_{c}(x, t)\right|^{2}+\left|\partial_{t} u_{c}(x, t)\right|^{2}=\int_{|y| \geq \lambda(t) R_{0}}|\vec{v}(x, t)|^{2} d y \underset{t \uparrow 1}{\longrightarrow} 0,
$$


because of the compactness of $\bar{K}$ and the fact that $\lambda(t) \rightarrow+\infty$ as $t \rightarrow 1$. Because of this fact, using the Remark backward in time, we have, for each $s \in[0,1)$, $R_{0}>0$,

$$
\lim _{t \uparrow 1} \int_{|x+x(t) / \lambda(t)| \geq \frac{3}{2} R_{0}+(t-s)}\left|\nabla u_{c}(x, s)\right|^{2}+\left|\partial_{t} u_{c}(x, s)\right|^{2}=0 .
$$

We next show that $|x(t) / \lambda(t)| \leq M, 0 \leq t<1$. If not, we can find $t_{n} \uparrow 1$ so that $\left|x\left(t_{n}\right) / \lambda\left(t_{n}\right)\right| \rightarrow+\infty$. Then, for $R>0,\{|x| \leq R\} \subset\left\{\left|x+x\left(t_{n}\right) / \lambda\left(t_{n}\right)\right| \geq\right.$ $\left.\frac{3}{2} R+t_{n}\right\}$ for $n$ large, so that, passing to the limit in $n$, for $s=0$, we obtain

$$
\int_{|x| \leq R}\left|\nabla u_{0, c}\right|^{2}+\left|u_{1, c}\right|^{2}=0
$$

a contradiction.

Finally, pick $t_{n} \uparrow 1$ so that $x\left(t_{n}\right) / \lambda\left(t_{n}\right) \rightarrow-\bar{x}$. Observe that, for every $\eta_{0}>0$, for $n$ large enough, for all $s \in[0,1),\left\{|x-\bar{x}| \geq 1+\eta_{0}-s\right\} \subset\left\{\left|x+x\left(t_{n}\right) / \lambda\left(t_{n}\right)\right| \geq\right.$ $\left.\frac{3}{2} R_{0}+\left(t_{n}-s\right)\right\}$, for some $R_{0}=R_{0}\left(\eta_{0}\right)>0$. From this we conclude that

$$
\int_{\left|x-x_{0}\right| \geq 1+\eta_{0}-s}|\nabla u(x, s)|^{2}+\left|\partial_{s} u(x, s)\right|^{2} d x=0,
$$

which gives the claim.

Note that, after translation, we can asume that $\bar{x}=0$. We next turn to a result which is fundamental for us to be able to treat non-radial data.

Theorem 8. Let $\left(u_{0, c}, u_{1, c}\right)$ be as in Proposition 4, with $\lambda(t), x(t)$ continuous. Assume that either $T_{+}\left(u_{0, c}, u_{1, c}\right)<\infty$ or $T_{+}\left(u_{0, c}, u_{1, c}\right)=+\infty, \lambda(t) \geq A_{0}>0$. Then

$$
\int \nabla u_{0, c} \cdot u_{1, c}=0
$$

In order to carry out the proof of this Theorem, a further linear estimate is needed:

Lemma 5. Let $w$ solve the linear wave equation

$$
\left\{\begin{array}{l}
\partial_{t}^{2} w-\triangle w=h \in L_{t}^{1} L_{x}^{2}\left(\mathbb{R}^{N+1}\right) \\
\left.w\right|_{t=0}=w_{0} \in \dot{H}^{1}\left(\mathbb{R}^{n}\right) \\
\left.\partial_{t} w\right|_{t=0}=w_{1} \in L^{2}\left(\mathbb{R}^{N}\right) .
\end{array}\right.
$$

Then, for $|a| \leq 1 / 4$, we have

$$
\begin{aligned}
& \sup _{t} \|\left(\nabla w\left(\frac{x_{1}-a t}{\sqrt{1-a^{2}}}, x^{\prime}, \frac{t-a x_{1}}{\sqrt{1-a^{2}}}\right), \partial_{t} w\right.\left.\left(\frac{x_{1}-a t}{\sqrt{1-a^{2}}}, x^{\prime}, \frac{t-a x_{1}}{\sqrt{1-a^{2}}}\right)\right) \|_{L^{2}\left(d x_{1} d x^{\prime}\right)} \\
& \leq C\left\{\left\|w_{0}\right\|_{\dot{H}^{1}}+\left\|w_{1}\right\|_{L^{2}}+\|h\|_{L_{t}^{1} L_{x}^{2}}\right\} .
\end{aligned}
$$


The simple proof is omitted; see [23] for the details. Note that if $u$ is a solution of (7), with maximal interval $I$ and $I^{\prime} \subset \subset I, u \in L_{I^{\prime}}^{(N+2) / N-2} L_{x}^{2(N+2) / N-2}$, and since $\frac{4}{N-2}+1=\frac{N+2}{N-2},|u|^{4 / N-2} u \in L_{I^{\prime}}^{1} L_{x}^{2}$. Thus, the conclusion of the Lemma applies, provided the integration is restricted to $\left(\frac{x_{1}-a t}{\sqrt{1-a^{2}}}, x^{\prime}, \frac{t-a x_{1}}{\sqrt{1-a^{2}}}\right) \in \mathbb{R}^{N} \times I^{\prime}$.

Sketch of the proof of the Theorem. Assume first that $T_{+}\left(u_{0, c}, u_{1, c}\right)=1$. Assume, to argue by contradiction, that (say) $\int \partial_{x_{1}}\left(u_{0, c}\right) u_{1, c}=\gamma>0$. Recall that, in this situation, supp $u_{c}, \partial_{t} u_{c} \subset B(0,1-t), 0<t<1$. For convenience, set $u(x, t)=$ $u_{c}(x, 1+t),-1<t<0$, which is supported in $B(0,|t|)$. For $0<a<1 / 4$, we consider the Lorentz transformation

$$
z_{a}\left(x_{1}, x^{\prime}, t\right)=u\left(\frac{x_{1}-a t}{\sqrt{1-a^{2}}}, x^{\prime}, \frac{t-a x_{1}}{\sqrt{1-a^{2}}}\right),
$$

and we fix our attention on $-1 / 2 \leq t<0$. In that region, the previous Lemma and the comment following show, in conjunction with the support property of $u$, that $z_{a}$ is a solution in the energy space of (7). An easy calculation shows that $\operatorname{supp} z_{a}(\cdot, t) \subset B(0,|t|)$, so that 0 is the final time of existence for $z_{a}$. A lengthy calculation shows that

$$
\lim _{a \downarrow 0} \frac{E\left(\left(z_{a}(\cdot,-1 / 2), \partial_{t} z_{a}(\cdot,-1 / 2)\right)\right)-E\left(\left(u_{0, c}, u_{1, c}\right)\right)}{a}=-\gamma
$$

and that, for some $t_{0} \in[-1 / 2,-1 / 4], \int\left|\nabla z_{a}\left(t_{0}\right)\right|^{2}<\int|\nabla W|^{2}$, for a small (by integration in $t_{0}$ and a change of variables, together with the variational estimates for $\left.u_{c}\right)$. But, since $E\left(\left(u_{0, c}, u_{1, c}\right)\right)=E_{c}$, for $a$ small this contradicts the definition of $E_{c}$, since the final time of existence of $z_{a}$ is finite.

In the case when $T_{+}\left(u_{0, c}, u_{1, c}\right)=+\infty, \lambda(t) \geq A_{0}>0$, the finiteness of the energy of $z_{a}$ is unclear, because of the lack of the support property. We instead do a renormalization. We first rescale $u_{c}$ and consider, for $R$ large, $u_{R}(x, t)=$ $R^{N-2 / 2} u_{c}(R x, R t)$, and for $a$ small,

$$
z_{a, R}\left(x_{1}, x^{\prime}, t\right)=u_{R}\left(\frac{x_{1}-a t}{\sqrt{1-a^{2}}}, x^{\prime}, \frac{t-a x_{1}}{\sqrt{1-a^{2}}}\right) .
$$

We assume, as before, that $\int \partial_{x_{1}}\left(u_{0, c}\right) u_{1, c}=\gamma>0$ and hope to obtain a contradiction. We prove, by integration in $t_{0} \in(1,2)$, that if $h\left(t_{0}\right)=\theta(x) z_{a, R}\left(x_{1}, x^{\prime}, t_{0}\right)$, with $\theta$ a fixed cut-off function, for some $a_{1}$ small and $R$ large, we have, for some $t_{0} \in(1,2)$, that

$$
E\left(\left(h\left(t_{0}\right), \partial_{t} h\left(t_{0}\right)\right)\right)<E_{c}-\frac{1}{2} \gamma a_{1}
$$

and

$$
\int\left|\nabla h\left(t_{0}\right)\right|^{2}<\int|\nabla W|^{2} .
$$


We then let $v$ be the solution of $(7)$ with data $h\left(\cdot, t_{0}\right)$. By the properties of $E_{c}$, we know that $\|v\|_{S(-\infty,+\infty)} \leq g\left(\frac{1}{2} \gamma a_{1}\right)$, for $R$ large. But, since $\left\|u_{c}\right\|_{S(0,+\infty)}=+\infty$, we have that

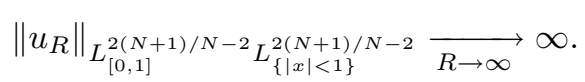

But, by finite speed of propagation, we have that $v=z_{a, R}$ on a large set and, after a change of variables to undo the Lorentz transformation, we reach a contradiction from these two facts.

From all this we see that, to prove Theorem 2, it suffices to show:

\section{Step 3: Rigidity Theorem.}

Theorem 9 (Rigidity). Assume that $E\left(\left(u_{0}, u_{1}\right)\right)<E((W, 0)), \int\left|\nabla u_{0}\right|^{2}<\int|\nabla W|^{2}$. Let $u$ be the corresponding solution of $(7)$, and let $I_{+}=\left[0, T_{+}\left(\left(u_{0}, u_{1}\right)\right)\right)$. Suppose that:

a) $\int \nabla u_{0} u_{1}=0$

b) There exist $x(t), \lambda(t), t \in\left[0, T_{+}\left(\left(u_{0}, u_{1}\right)\right)\right)$ such that

$$
K=\left\{v(x, t)=\left(\frac{1}{\lambda(t)^{N-2 / 2}} u_{c}\left(\frac{x-x(t)}{\lambda(t)}, t\right), \frac{1}{\lambda(t)^{N / 2}} \partial_{t} u_{c}\left(\frac{x-x(t)}{\lambda(t)}, t\right)\right): t \in I_{+}\right\}
$$

has compact closure in $\dot{H}^{1} \times L^{2}$.

c) $x(t), \lambda(t)$ are continuous, $\lambda(t)>0$. If $T_{+}\left(u_{0}, u_{1}\right)<\infty$, we have $\lambda(t) \geq$ $C /\left(T_{+}-t\right)$, supp $u, \partial_{t} u \subset B\left(0, T_{+}-t\right)$, and if $T_{+}\left(u_{0}, u_{1}\right)=+\infty$, we have $x(0)=0, \lambda(0)=1, \lambda(t) \geq A_{0}>0$.

Then $T_{+}\left(u_{0}, u_{1}\right)=+\infty, u \equiv 0$.

Clearly this Rigidity Theorem provides the contradiction that concludes the proof of Theorem 2.

Proof of the Rigidity Theorem. For the proof we need some known identities (see [42], [23]).

Lemma 6. Let

$$
r(R)=r(t, R)=\int_{|x| \geq R}\left\{|\nabla u|^{2}+\left|\partial_{t} u\right|^{2}+|u|^{2^{*}}+\frac{|u|^{2}}{|x|^{2}}\right\} d x .
$$

Let $u$ be a solution of $(7), t \in I, \phi_{R}(x)=\phi(x / R), \psi_{R}(x)=x \phi(x / R)$, where $\phi$ is in $C_{0}^{\infty}\left(B_{2}\right), \phi \equiv 1$ on $|x| \leq 1$. Then:

i) $\partial_{t}\left(\int \psi_{R} \nabla u \partial_{t} u\right)=-\frac{N}{2} \int\left(\partial_{t} u\right)^{2}+\frac{N-2}{2} \int\left[|\nabla u|^{2}-|u|^{2^{*}}\right]+\mathcal{O}(r(R))$. 
ii) $\partial_{t}\left(\int \phi_{R} \nabla u \partial_{t} u\right)=\int\left(\partial_{t} u\right)^{2}-\int|\nabla u|^{2}+\int|u|^{2^{*}}+\mathcal{O}(r(R))$.

iii) $\partial_{t}\left(\int \psi_{R}\left\{\frac{1}{2}|\nabla u|^{2}+\frac{1}{2}\left(\partial_{t} u\right)^{2}-\frac{1}{2^{*}}|u|^{2^{*}}\right\}\right)=-\int \nabla u \partial_{t} u+\mathcal{O}(r(R))$.

We start out the proof of case $1, T_{+}\left(\left(u_{0}, u_{1}\right)\right)=+\infty$, by observing that, if $\left(u_{0}, u_{1}\right) \neq(0,0)$ and $E=E\left(\left(u_{0}, u_{1}\right)\right)$, then, from our variational estimates, $E>0$ and

$$
\sup _{t>0}\left\|\left(\nabla u(t), \partial_{t} u(t)\right)\right\|_{\dot{H}^{1} \times L^{2}} \leq C E
$$

We also have

$$
\int|\nabla u(t)|^{2}-|u(t)|^{2^{*}} \geq C \int|\nabla u(t)|^{2}, \quad t>0
$$

and

$$
\frac{1}{2} \int\left(\partial_{t} u(t)\right)^{2}+\frac{1}{2} \int\left[|\nabla u(t)|^{2}-|u(t)|^{2^{*}}\right] \geq C E, \quad t>0
$$

The compactness of $\bar{K}$ and the fact that $\lambda(t) \geq A_{0}>0$ show that, given $\epsilon>0$, we can find $R_{0}(\epsilon)>0$ so that, for all $t>0$, we have

$$
\int_{\left|x+\frac{x(t)}{\lambda(t)}\right| \geq R(\epsilon)}\left|\partial_{t} u\right|^{2}+|\nabla u|^{2}+\frac{|u|^{2}}{|x|^{2}}+|u|^{2^{*}} \leq \epsilon E .
$$

The proof of this case is accomplished through two lemmas.

Lemma 7. There exist $\epsilon_{1}>0, C>0$ such that, if $0<\epsilon<\epsilon_{1}$, if $R>2 R_{0}(\epsilon)$, there exists $t_{0}=t_{0}(R, \epsilon)$ with $0<t_{0} \leq C R$, such that for $0<t<t_{0}$, we have $\left|\frac{x(t)}{\lambda(t)}\right|<R-R_{0}(\epsilon)$ and $\left|\frac{x(t)}{\lambda(t)}\right|=R-R_{0}(\epsilon)$.

Note that in the radial case, since we can take $x(t) \equiv 0$, a contradiction follows directly from Lemma 7 . This will be the analog of the local virial identity proof for the corresponding case of (4). For the non-radial case we also need:

Lemma 8. There exist $\epsilon_{2}>0, R_{1}(\epsilon)>0, C_{0}>0$, so that if $R>R_{1}(\epsilon)$, for $0<\epsilon<\epsilon_{2}$, we have $t_{0}(R, \epsilon) \geq C_{0} R / \epsilon$, where $t_{0}$ is as in Lemma 7 .

From Lemma 7 and Lemma 8 we have, for $0<\epsilon<\epsilon_{1}, R>2 R_{0}(\epsilon)$, $t_{0}(R, \epsilon) \leq C R$, while for $0<\epsilon<\epsilon_{2}, R>R_{1}(\epsilon), t_{0}(R, \epsilon) \geq C_{0} R / \epsilon$. This is clearly a contradiction for $\epsilon$ small.

Proof of Lemma 7. Since $x(0)=0, \lambda(0)=1$; if not, we have for all $0<t<C R$, with $C$ large, that $\left|\frac{x(t)}{\lambda(t)}\right|<R-R_{0}(\epsilon)$. Let

$$
z_{R}(t)=\int \psi_{R} \nabla u \partial_{t} u+\left(\frac{N}{2}-\frac{1}{2}\right) \int \phi_{R} u \partial_{t} u
$$


Then

$$
z_{R}^{\prime}(t)=-\frac{1}{2} \int\left(\partial_{t} u\right)^{2}-\frac{1}{2} \int\left[|\nabla u|^{2}-|u|^{2^{*}}\right]+\mathcal{O}(r(R))
$$

But, for $|x|>R, 0<t<C R$, we have $\left|x+\frac{x(t)}{\lambda(t)}\right| \geq R_{0}(\epsilon)$ so that $|r(R)| \leq \tilde{C} \epsilon E$. Thus, for $\epsilon$ small, $z_{R}^{\prime}(t) \leq-\tilde{\tilde{C}} E / 2$. By our variational estimates, we also have $\left|z_{R}(T)\right| \leq C_{1} R E$. Integrating in $t$ we obtain $C R \tilde{\widetilde{C}} E / 2 \leq 2 C_{1} R E$, which is a contradiction for $C$ large.

Proof of Lemma 8. For $0 \leq t \leq t_{0}$, set

$$
y_{R}(t)=\int \psi_{R}\left\{\frac{1}{2}\left(\partial_{t} u\right)^{2}+\frac{1}{2}|\nabla u|^{2}-\frac{1}{2^{*}}|u|^{2^{*}}\right\} .
$$

For $|x|>R,\left|x+\frac{x(t)}{\lambda(t)}\right| \geq R_{0}(\epsilon)$, so that, since $\int \nabla u_{0} u_{1}=0=\int \nabla u(t) \partial_{t} u(t)$, $y^{\prime}(R)=\mathcal{O}(r(R))$, and hence $\left|y_{R}\left(t_{0}\right)-y_{R}(0)\right| \leq \tilde{C} \epsilon E t_{0}$. However,

$$
\left|y_{R}(0)\right| \leq \tilde{C} R_{0}(\epsilon) E+\mathcal{O}\left(R r\left(R_{0}(\epsilon)\right)\right) \leq \tilde{C} E\left[R_{0}(\epsilon)+\epsilon R\right]
$$

Also,

$$
\begin{aligned}
\left|y_{R}\left(t_{0}\right)\right| & \geq\left|\int_{\left|x+\frac{x\left(t_{0}\right)}{\lambda\left(t_{0}\right)}\right| \leq R_{0}(\epsilon)} \psi_{R}\left\{\frac{1}{2}\left(\partial_{t} u\right)^{2}+\frac{1}{2}|\nabla u|^{2}-\frac{1}{2^{*}}|u|^{2^{*}}\right\}\right|- \\
& -\left|\int_{\left|x+\frac{x\left(t_{0}\right)}{\lambda\left(t_{0}\right)}\right|>R_{0}(\epsilon)} \psi_{R}\left\{\frac{1}{2}\left(\partial_{t} u\right)^{2}+\frac{1}{2}|\nabla u|^{2}-\frac{1}{2^{*}}|u|^{2^{*}}\right\}\right| .
\end{aligned}
$$

In the first integral, $|x| \leq R$, so that $\psi_{R}(x)=x$. The second integral is bounded by $M R \epsilon E$. Thus,

$$
\left|y_{R}\left(t_{0}\right)\right| \geq\left|\int_{\left|x+\frac{x\left(t_{0}\right)}{\lambda\left(t_{0}\right)}\right| \leq R_{0}(\epsilon)} x\left\{\frac{1}{2}\left(\partial_{t} u\right)^{2}+\frac{1}{2}|\nabla u|^{2}-\frac{1}{2^{*}}|u|^{2^{*}}\right\}\right|-M R \epsilon E .
$$

The integral on the right equals

$$
\begin{aligned}
& -\frac{x\left(t_{0}\right)}{\lambda\left(t_{0}\right)} \int_{\left|x+\frac{x\left(t_{0}\right)}{\lambda\left(t_{0}\right)}\right| \leq R_{0}(\epsilon)}\left\{\frac{1}{2}\left(\partial_{t} u\right)^{2}+\frac{1}{2}|\nabla u|^{2}-\frac{1}{2^{*}}|u|^{2^{*}}\right\}+ \\
& +\int_{\left|x+\frac{x\left(t_{0}\right)}{\lambda\left(t_{0}\right)}\right| \leq R_{0}(\epsilon)}\left(x+\frac{x\left(t_{0}\right)}{\lambda\left(t_{0}\right)}\right)\left\{\frac{1}{2}\left(\partial_{t} u\right)^{2}+\frac{1}{2}|\nabla u|^{2}-\frac{1}{2^{*}}|u|^{2^{*}}\right\},
\end{aligned}
$$

so that its absolute value is greater than or equal to

$$
\left(R_{0}-R_{0}(\epsilon)\right) E-\tilde{C}\left(R-R_{0}(\epsilon)\right) \epsilon E-\tilde{C} R_{0}(\epsilon) E .
$$


Thus,

$$
\left|y_{R}\left(t_{0}\right)\right| \geq E\left(R-R_{0}(\epsilon)\right)[1-\tilde{C} \epsilon]-\tilde{C} R_{0}(\epsilon) E-M R \epsilon E \geq E R / 4,
$$

for $R$ large, $\epsilon$ small. But then $E R / 4-\tilde{C} E\left[R_{0}(\epsilon)+\epsilon R\right] \leq \tilde{C} \epsilon E t_{0}$, which yields the Lemma for $\epsilon$ small, $R$ large.

We next turn to the case $2, T_{+}\left(\left(u_{0}, u_{1}\right)\right)=1$, with $\operatorname{supp} u, \partial_{t} u \subset B(0,1-t)$, $\lambda(t) \geq C / 1-t$. For $(7)$ we cannot use the conservation of the $L^{2}$ norm as in the (4) case and a new approach is needed. The first step is:

Lemma 9. Let $u$ be as in the Rigidity Theorem, with $T_{+}\left(\left(u_{0}, u_{1}\right)\right)=1$. Then there exists $C>0$ so that $\lambda(t) \leq C / 1-t$.

Proof. If not, we can find $t_{n} \uparrow 1$ so that $\lambda\left(t_{n}\right)\left(1-t_{n}\right) \rightarrow+\infty$. Let

$$
z(t)=\int x \nabla u \partial_{t} u+\left(\frac{N}{2}-\frac{1}{2}\right) \int u \partial_{t} u,
$$

where we recall that $z$ is well defined since $\operatorname{supp} u, \partial_{t} u \subset B(0,1-t)$. Then, for $0<t<1$, we have

$$
z^{\prime}(t)=-\frac{1}{2} \int\left(\partial_{t} u\right)^{2}-\frac{1}{2} \int|\nabla u|^{2}-|u|^{2^{*}}
$$

By our variational estimates, $E\left(\left(u_{0}, u_{1}\right)\right)=E>0$ and

$$
\sup _{0<t<1}\left\|\left(u(t), \partial_{t} u\right)\right\|_{\dot{H}^{1} \times L^{2}} \leq C E
$$

and $z^{\prime}(t) \leq-C E$, for $0<t<1$. From the support properties of $u$, it is easy to see that $\lim _{t \uparrow 1} z(t)=0$, so that, integrating in $t$, we obtain

$$
z(t) \geq C E(1-t), \quad 0 \leq t<1 .
$$

We will next show that $z\left(t_{n}\right) /\left(1-t_{n}\right) \underset{n \rightarrow \infty}{\longrightarrow} 0$, yielding a contradiction. Because $\int \nabla u(t) \partial_{t} u(t)=0,0<t<1$, we have

$$
\frac{z\left(t_{n}\right)}{1-t_{n}}=\int \frac{\left(x+x\left(t_{n}\right) / \lambda\left(t_{n}\right)\right) \nabla u \partial_{t} u}{1-t_{n}}+\left(\frac{N}{2}-\frac{1}{2}\right) \int \frac{u \partial_{t} u}{1-t_{n}} .
$$

Note that, for $\epsilon>0$ given, we have

$$
\int_{\left|x+\frac{x\left(t_{n}\right)}{\lambda\left(t_{n}\right)}\right| \leq \epsilon\left(1-t_{n}\right)}\left|x+\frac{x\left(t_{n}\right)}{\lambda\left(t_{n}\right)}\right|\left|\nabla u\left(t_{n}\right)\right|\left|\partial_{t} u\left(t_{n}\right)\right|+\left|u\left(t_{n}\right)\right|\left|\partial_{t} u\left(t_{n}\right)\right| \leq C \epsilon E\left(1-t_{n}\right) .
$$

Next we will show that $\left|x\left(t_{n}\right) / \lambda\left(t_{n}\right)\right| \leq 2\left(1-t_{n}\right)$. If not, $B\left(-x\left(t_{n}\right) / \lambda\left(t_{n}\right),(1-\right.$ $\left.\left.t_{n}\right)\right) \cap B\left(0,\left(1-t_{n}\right)\right)=\emptyset$, so that

$$
\int_{B\left(-x\left(t_{n}\right) / \lambda\left(t_{n}\right),\left(1-t_{n}\right)\right)}\left|\nabla u\left(t_{n}\right)\right|^{2}+\left|\partial_{t} u\left(t_{n}\right)\right|^{2}=0
$$


while

$$
\begin{aligned}
\int_{\left|x+\frac{x\left(t_{n}\right)}{\lambda\left(t_{n}\right)}\right| \geq\left(1-t_{n}\right)}\left|\nabla u\left(t_{n}\right)\right|^{2}+\left|\partial_{t} u\left(t_{n}\right)\right|^{2} & =\int_{|y| \geq \lambda\left(t_{n}\right)\left(1-t_{n}\right)}\left|\nabla u\left(\frac{y-x\left(t_{n}\right)}{\lambda\left(t_{n}\right)}, t_{n}\right)\right|^{2}+ \\
& +\left|\partial_{t} u\left(\frac{y-x\left(t_{n}\right)}{\lambda\left(t_{n}\right)}, t_{n}\right)\right|^{2} \frac{d y}{\lambda\left(t_{n}\right)^{N}} \underset{n \rightarrow \infty}{\longrightarrow} 0,
\end{aligned}
$$

which contradicts $E>0$. Then

$$
\begin{aligned}
& \frac{1}{1-t_{n}} \int_{\left|x+\frac{x\left(t_{n}\right)}{\lambda\left(t_{n}\right)}\right| \geq \epsilon\left(1-t_{n}\right)}\left|x+\frac{x\left(t_{n}\right)}{\lambda\left(t_{n}\right)}\right| \nabla u\left(t_{n}\right)|| \partial_{t} u\left(t_{n}\right) \mid \leq \\
& \leq 3 \int_{\left|x+\frac{x\left(t_{n}\right)}{\lambda\left(t_{n}\right)}\right| \geq \epsilon\left(1-t_{n}\right)}\left|\nabla u\left(t_{n}\right)\right|\left|\partial_{t} u\left(t_{n}\right)\right|= \\
& =3 \int_{|y| \geq \epsilon\left(1-t_{n}\right) \lambda\left(t_{n}\right)}\left|\nabla u\left(\frac{y-x\left(t_{n}\right)}{\lambda\left(t_{n}\right)}, t_{n}\right)\right|\left|\partial_{t} u\left(\frac{y-x\left(t_{n}\right)}{\lambda\left(t_{n}\right)}, t_{n}\right)\right| \frac{d y}{\lambda\left(t_{n}\right)^{N}} \\
& \quad \underset{n \rightarrow \infty}{\longrightarrow} 0
\end{aligned}
$$

because of the compactness of $\bar{K}$ and the fact that $\lambda\left(t_{n}\right)\left(1-t_{n}\right) \rightarrow \infty$. Arguing similarly for $\int \frac{u \partial_{t} u}{1-t_{n}}$, using Hardy's inequality (centered at $-x\left(t_{n}\right) / \lambda\left(t_{n}\right)$ ), the proof is concluded.

Proposition 5. Let $u$ be as in the Rigidity Theorem, with $T_{+}\left(\left(u_{0}, u_{1}\right)\right)=1, \operatorname{supp} u$, $\partial_{t} u \subset B(0,1-t)$. Then

$$
K=\left((1-t)^{N-2 / 2} u((1-t) x, t),(1-t)^{N-2 / 2} \partial_{t} u((1-t) x, t)\right)
$$

is precompact in $\dot{H}^{1}\left(\mathbb{R}^{N}\right) \times L^{2}\left(\mathbb{R}^{N}\right)$.

Proof.

$\left\{\vec{v}(x, t)=(1-t)^{\frac{N}{2}}\left(\nabla u((1-t)(x-x(t)), t), \partial_{t} u((1-t)(x-x(t)), t)\right), 0 \leq t<1\right\}$

has compact closure in $L^{2}\left(\mathbb{R}^{N}\right)^{N+1}$, since we have $c_{0} \leq(1-t) \lambda(t) \leq c_{1}$ and if $\bar{K}$ is compact in $L^{2}\left(\mathbb{R}^{N}\right)^{N+1}$,

$$
K_{1}=\left\{\lambda^{N / 2} \vec{v}(\lambda x): \vec{v} \in K, c_{0} \leq \lambda \leq c_{1}\right\}
$$

also has $\bar{K}_{1}$ compact. Let now

$$
\tilde{v}(x, t)=(1-t)^{N / 2}\left(\nabla u((1-t) x, t), \partial_{t} u((1-t) x, t)\right),
$$

so that $\tilde{v}(x, t)=\vec{v}(x+x(t), t)$. Since $\operatorname{supp} \vec{v}(\cdot, t) \subset\{x:|x-x(t)| \leq 1\}$ and $E>0$, the fact that $\{\vec{v}(\cdot, t)\}$ is compact implies that $|x(t)| \leq M$. But if $K_{2}=\{\vec{v}(x+$ $\left.\left.x_{0}, t\right):\left|x_{0}\right| \leq M\right\}$, then $\bar{K}_{2}$ is compact, giving the Proposition. 
At this point we introduce a new idea, inspired by the works of Giga-Kohn [12] in the parabolic case and Merle-Zaag [36] in the hyperbolic case, who studied the equations $\left(\partial_{t}^{2}-\triangle\right) u-|u|^{p-1} u=0$, for $1<p<\frac{4}{N-1}+1$, in the radial case. In our case, $p=\frac{4}{N-2}+1>\frac{4}{N-1}+1$. We thus introduce self-similar variables. Thus, we set $y=x / 1-t, s=\log 1 / 1-t$ and define

$$
w(y, s ; 0)=(1-t)^{N-2 / 2} u(x, t)=e^{-s(N-2) / 2} u\left(e^{-s} y, 1-e^{-s}\right),
$$

which is defined for $0 \leq s<\infty$ with $\operatorname{supp} w(\cdot, s ; 0) \subset\{|y| \leq 1\}$. We will also consider, for $\delta>0, u_{\delta}(x, t)=u(x, t+\delta)$ which also solves $(7)$ and its corresponding $w$, which we will denote by $w(y, s ; \delta)$. Thus, we set $y=x / 1+\delta-t, s=\log 1 / 1+\delta-t$ and

$$
w(y, s ; \delta)=(1+\delta-t)^{N-2 / 2} u(x, t)=e^{-s(N-2) / 2} u\left(e^{-s} y, 1+\delta-e^{-s}\right) .
$$

Here $w(y, s ; \delta)$ is defined for $0 \leq s<-\log \delta$ and we have

$$
\operatorname{supp} w(\cdot, s ; \delta) \subset\left\{|y| \leq \frac{e^{-s}-\delta}{e^{-s}}=\frac{1-t}{1+\delta-t} \leq 1-\delta\right\} .
$$

The $w$ solve, where they are defined, the equation

$$
\begin{aligned}
\partial_{s}^{2} w & =\frac{1}{\rho} \operatorname{div}(\rho \nabla w-\rho(y \cdot \nabla w) y)-\frac{N(N-2)}{4} w+ \\
& +|w|^{4 / N-2} w-2 y \cdot \nabla \partial_{s} w-(N-1) \partial_{s} w
\end{aligned}
$$

where $\rho(y)=\left(1-|y|^{2}\right)^{-1 / 2}$.

Note that the elliptic part of this operator degenerates. In fact,

$$
\frac{1}{\rho} \operatorname{div}(\rho \nabla w-\rho(y \cdot \nabla w) y)=\frac{1}{\rho} \operatorname{div}(\rho(I-y \otimes y) \nabla w),
$$

which is elliptic with smooth coefficients for $|y|<1$, but degenerates at $|y|=1$.

Here are some straightforward bounds on $w(\cdot ; \delta)(\delta>0): w \in H_{0}^{1}\left(B_{1}\right)$ with

$$
\int_{B_{1}}|\nabla w|^{2}+\left|\partial_{s} w\right|^{2}+|w|^{2^{*}} \leq C
$$

Moreover, by Hardy's inequality for $H_{0}^{1}\left(B_{1}\right)$ functions [6],

$$
\int_{B_{1}} \frac{|w(y)|^{2}}{\left(1-|y|^{2}\right)^{2}} \leq C \text {. }
$$

These bounds are uniform in $\delta>0,0<s<-\log \delta$. Next, following [36], we introduce an energy, which will provide us with a Lyapunov functional for $w$.

$$
\begin{aligned}
\tilde{E}(w(s ; \delta)) & =\int_{B_{1}} \frac{1}{2}\left\{\left(\partial_{s} w\right)^{2}+|\nabla w|^{2}-(y \cdot \nabla w)^{2}\right\} \frac{d y}{\left(1-|y|^{2}\right)^{1 / 2}}+ \\
& +\int_{B_{1}}\left\{\frac{N(N-2)}{8} w^{2}-\frac{N-2}{2 N}|w|^{2^{*}}\right\} \frac{d y}{\left(1-|y|^{2}\right)^{1 / 2}}
\end{aligned}
$$


Note that this is finite for $\delta>0$. We have:

Lemma 10. For $\delta>0,0<s_{1}<s_{2}<\log 1 / \delta$,

i) $\tilde{E}\left(w\left(s_{2}\right)\right)-\tilde{E}\left(w\left(s_{1}\right)\right)=\int_{s_{1}}^{s_{2}} \int_{B_{1}} \frac{\left(\partial_{s} w\right)^{2}}{\left(1-|y|^{2}\right)^{3 / 2}} d s d y$, so that $\tilde{E}$ is increasing.

ii) $\left.\frac{1}{2} \int_{B_{1}}\left[\left(\partial_{s} w\right) \cdot w-\frac{1+N}{2} w^{2}\right] \frac{d y}{\left(1-|y|^{2}\right)^{1 / 2}}\right|_{s_{1}} ^{s_{2}}=$

$$
\begin{aligned}
& =-\int_{s_{1}}^{s_{2}} \tilde{E}(w(s)) d s+\frac{1}{N} \int_{s_{1}}^{s_{2}} \int_{B_{1}} \frac{|w|^{2^{*}}}{\left(1-|y|^{2}\right)^{1 / 2}} d s d y+ \\
& +\int_{s_{1}}^{s_{2}} \int_{B_{1}}\left\{\left(\partial_{s} w\right)^{2}+\partial_{s} w y \cdot \nabla w+\frac{\partial_{s} w w|y|^{2}}{1-|y|^{2}}\right\} \frac{d y}{\left(1-|y|^{2}\right)^{1 / 2}} .
\end{aligned}
$$

iii) $\lim _{s \rightarrow \log 1 / \delta} \tilde{E}(w(s))=E\left(\left(u_{0}, u_{1}\right)\right)=E$, so that, by i), $\tilde{E}(w(s)) \leq E$ for $0 \leq$ $s<\log 1 / \delta$.

The proof is computational; see [23]. Our first improvement over this is:

Lemma 11. $\int_{0}^{1} \int_{B_{1}} \frac{\left(\partial_{s} w\right)^{2}}{1-|y|^{2}} d y d s \leq C \log 1 / \delta$.

Proof. Notice that

$$
\begin{aligned}
-2 \int \frac{\left(\partial_{s} w\right)^{2}}{1-|y|^{2}} & =\frac{d}{d s}\left\{\int \left[\frac{1}{2}\left(\partial_{s} w\right)^{2}+\frac{1}{2}\left(|\nabla w|^{2}-(y \cdot \nabla w)^{2}\right)+\right.\right. \\
& \left.+\frac{(N-2) N}{8} w^{2}-\frac{N-2}{2 N}|w|^{2^{*}}\right]\left[-\log \left(1-|y|^{2}\right)\right] d y+ \\
& +\int\left[\log \left(1-|y|^{2}\right)+2\right] y \cdot \nabla w \partial_{s} w-\log \left(1-|y|^{2}\right)\left(\partial_{s} w\right)^{2}- \\
& -2 \int\left(\partial_{s} w\right)^{2}
\end{aligned}
$$

We next integrate in $s$, between 0 and 1 , and drop the next to last term by sign. The proof is finished by using Cauchy-Schwartz and the support property of $w(\cdot ; \delta)$.

Corollary 4. a) $\int_{0}^{1} \int_{B_{1}} \frac{|w|^{2^{*}}}{\left(1-|y|^{2}\right)^{1 / 2}} d y d s \leq C(\log 1 / \delta)^{1 / 2}$.

b) $\tilde{E}(w(1)) \geq-C(\log 1 / \delta)^{1 / 2}$. 
Proof. Part a) follows from ii), iii) above, Cauchy-Schwartz and the previous Lemma. Note that we obtain the power $1 / 2$ on the right hand side by CauchySchwartz. Part b) follows from i) and the fact that

$$
\int_{0}^{1} \tilde{E}(w(s)) d s \geq-C(\log 1 / \delta)^{1 / 2},
$$

which is a consequence of the definition of $\tilde{E}$ and a).

Our next improvement is:

Lemma 12. $\int_{1}^{\log 1 / \delta} \int_{B_{1}} \frac{\left(\partial_{s} w\right)^{2}}{\left(1-|y|^{2}\right)^{3 / 2}} \leq C(\log 1 / \delta)^{1 / 2}$.

Proof. Use i), iii) and the bound b) in the Corollary.

Corollary 5. There exists $\bar{s}_{\delta} \in\left(1,(\log 1 / \delta)^{3 / 4}\right)$ such that

$$
\int_{\bar{s}_{\delta}}^{\bar{s}_{\delta}+(\log 1 / \delta)^{1 / 8}} \int_{B_{1}} \frac{\left(\partial_{s} w\right)^{2}}{\left(1-|y|^{2}\right)^{3 / 2}} \leq \frac{C}{(\log 1 / \delta)^{1 / 8}} .
$$

Proof. Split $\left(1,(\log 1 / \delta)^{3 / 4}\right)$ into disjoint intervals of length $(\log 1 / \delta)^{1 / 8}$. Their number is $(\log 1 / \delta)^{5 / 8}$ and $\frac{5}{8}-\frac{1}{8}=\frac{1}{2}$.

Note that, in the Corollary, the length of the $s$ interval tends to infinity, while the bound goes to zero. It is easy to see that if $\bar{s}_{\delta} \in\left(1,(\log 1 / \delta)^{3 / 4}\right)$, and $\bar{s}_{\delta}=-\log \left(1+\delta-\bar{t}_{\delta}\right)$, then

$$
\left|\frac{1-\bar{t}_{\delta}}{1+\delta-\bar{t}_{\delta}}-1\right| \leq C \delta^{1 / 4}
$$

which goes to 0 with $\delta$. From this and the compactness of $\bar{K}$, we can find $\delta_{j} \rightarrow 0$, so that $w\left(y, \bar{s}_{\delta_{j}}+s ; \delta_{j}\right)$ converges, for $s \in[0, S]$ to $w^{*}(y, s)$ in $C\left([0, S] ; \dot{H}_{0}^{1} \times L^{2}\right)$, and $w^{*}$ solves our self-similar equation in $B_{1} \times[0, S]$. The previous Corollary shows that $w^{*}$ must be independent of $s$. Also, the fact that $E>0$ and our coercivity estimates show that $w^{*} \not \equiv 0$. (See [23] for the details.) Thus, $w^{*} \in H_{0}^{1}\left(B_{1}\right)$ solves the (degenerate) elliptic equation

$$
\begin{gathered}
\frac{1}{\rho} \operatorname{div}\left(\rho \nabla w^{*}-\rho\left(y \cdot \nabla w^{*}\right) y\right)-\frac{N(N-2)}{4} w^{*}+\left|w^{*}\right|^{4 / N-2} w^{*}=0, \\
\rho(y)=\left(1-|y|^{2}\right)^{-1 / 2} .
\end{gathered}
$$

We next point out that $w^{*}$ satisfies the additional (crucial) estimates:

$$
\int_{B_{1}} \frac{\left|w^{*}\right|^{2^{*}}}{\left(1-|y|^{2}\right)^{1 / 2}}+\int_{B_{1}} \frac{\left[\left|\nabla w^{*}\right|^{2}-\left(y \cdot \nabla w^{*}\right)^{2}\right]}{\left(1-|y|^{2}\right)^{1 / 2}}<\infty .
$$


Indeed, for the first estimate it suffices to show that, uniformly in $j$ large, we have

$$
\int_{\bar{s}_{\delta_{j}}}^{\bar{s}_{\delta_{j}}+\delta} \int_{B_{1}} \frac{\left|w\left(y, s ; \delta_{j}\right)\right|^{2^{*}}}{\left(1-|y|^{2}\right)^{1 / 2}} d y d s \leq C
$$

which follows from ii) above, together with the choice of $\bar{s}_{\delta_{j}}$, by the Corollary, Cauchy-Schwartz and iii). The proof of the second estimate follows from the first one, iii) and the formula for $\tilde{E}$.

The conclusion of the proof is obtained by showing that a $w^{*}$ in $H_{0}^{1}\left(B_{1}\right)$, solving the degenerate elliptic equation with the additional bounds above, must be zero. This will follow from a unique continuation argument. Recall that, for $|y| \leq 1-\eta_{0}, \eta_{0}>0$, the linear operator is uniformly eliptic, with smooth coefficients and that the non-linearity is critical. An argument of Trudinger's [51] shows that $w^{*}$ is bounded on $\left\{|y| \leq 1-\eta_{0}\right\}$ for each $\eta_{0}>0$. Thus, if we show that $w^{*} \equiv 0$ near $|y|=1$, the standard Carleman unique continuation principle [19] will show that $w^{*} \equiv 0$.

Near $|y|=1$, our equation is modeled (in variables $z \in \mathbb{R}^{N-1}, r \in \mathbb{R}, r>0$, near $r=0$ ) by

$$
r^{1 / 2} \partial_{r}\left(r^{1 / 2} \partial_{r} w^{*}\right)+\triangle_{z} w^{*}+c w^{*}+\left|w^{*}\right|^{4 / N-2} w^{*}=0 .
$$

Our information on $w^{*}$ translates into $w^{*} \in H_{0}^{1}((0,1] \times(|z|<1))$ and our crucial additional estimates are:

$$
\int_{0}^{1} \int_{|z|<1}\left|w^{*}(r, z)\right|^{2^{*}} \frac{d r}{r^{1 / 2}} d z+\int_{0}^{1} \int_{|z|<1}\left|\nabla_{z} w^{*}(r, z)\right|^{2} \frac{d r}{r^{1 / 2}} d z<\infty .
$$

To conclude, we take advantage of the degeneracy of the equation. We "desingularize" the problem by letting $r=a^{2}$, setting $v(a, z)=w^{*}\left(a^{2}, z\right)$, so that $\partial_{a} v(a, z)=2 r^{1 / 2} \partial_{r} w^{*}(r, z)$. Our equation becomes:

$$
\partial_{a}^{2} v+\triangle_{z} v+c v+|v|^{4 / N-2} v=0, \quad 0<a<1, \quad|z|<1,\left.\quad v\right|_{a=0}=0,
$$

and our bounds give:

$$
\begin{gathered}
\int_{0}^{1} \int_{|z|<1}\left|\nabla_{z} v(a, z)\right|^{2} d a d z=\int_{0}^{1} \int_{|z|<1}\left|\nabla_{z} w^{*}(r, z)\right|^{2} \frac{d r}{r^{1 / 2}} d z<\infty \\
\int_{0}^{1} \int_{|z|<1}\left|\partial_{a} v(a, z)\right|^{2} \frac{d a}{a} d z=\int_{0}^{1} \int_{|z|<1}\left|\partial_{r} w^{*}(r, z)\right|^{2} d r d z<\infty
\end{gathered}
$$

Thus, $v \in H_{0}^{1}\left((0,1] \times B_{1}\right)$, but in addition $\left.\partial_{a} v(a, z)\right|_{a=0} \equiv 0$. We then extend $v$ by 0 to $a<0$ and see that the extension is an $H^{1}$ solution to the same equation. By Trudinger's argument, it is bounded. But since it vanishes for $a<0$, by Carleman's unique continuation theorem, $v \equiv 0$. Hence, $w^{*} \equiv 0$, giving our contradiction. 


\section{Bibliography}

[1] T. Aubin. Équations différentielles non linéaires et problème de Yamabe concernant la courbure scalaire. J. Math. Pures Appl. (9), 55(3):269-296, 1976.

[2] H. Bahouri and P. Gérard. High frequency approximation of solutions to critical nonlinear wave equations. Amer. J. Math., 121(1):131-175, 1999.

[3] H. Bahouri and J. Shatah. Decay estimates for the critical semilinear wave equation. Ann. Inst. H. Poincaré Anal. Non Linéaire, 15(6):783-789, 1998.

[4] J. Bourgain. Global wellposedness of defocusing critical nonlinear Schrödinger equation in the radial case. J. Amer. Math. Soc., 12(1):145-171, 1999.

[5] H. Brézis and J.-M. Coron. Convergence of solutions of $H$-systems or how to blow bubbles. Arch. Rational Mech. Anal., 89(1):21-56, 1985.

[6] H. Brézis and M. Marcus. Hardy's inequalities revisited. Ann. Scuola Norm. Sup. Pisa Cl. Sci. (4), 25(1-2):217-237 (1998), 1997. Dedicated to Ennio De Giorgi.

[7] T. Cazenave and F. B. Weissler. The Cauchy problem for the critical nonlinear Schrödinger equation in $H^{s}$. Nonlinear Anal., 14(10):807-836, 1990.

[8] J. Colliander, M. Keel, G. Staffilani, H. Takaoka, and T. Tao. Global wellposedness and scattering for the energy-critical nonlinear Schrödinger equation in $\mathbb{R}^{3}$. Annals of Math., to appear.

[9] R. Côte, C. Kenig, and F. Merle. Scattering below critical energy for the radial 4D Yang-Mills equation and for the 2D corotational wave map system, 2007. arXiv:math.AP/0709.3222v1. Comm. Math. Phys., to appear.

[10] T. Duyckaerts, J. Holmer, and S. Roudenko. Scattering for the non-radial 3D cubic nonlinear Schrödinger equation, 2007. arXiv:math.AP/0710.3630.

[11] L. Escauriaza, G. A. Serëgin, and V. Sverak. $L_{3, \infty}$-solutions of Navier-Stokes equations and backward uniqueness. Russ. Math. Surv., 58(2):211-250, 2003. 
[12] Y. Giga and R. V. Kohn. Nondegeneracy of blowup for semilinear heat equations. Comm. Pure Appl. Math., 42(6):845-884, 1989.

[13] J. Ginibre, A. Soffer, and G. Velo. The global Cauchy problem for the critical nonlinear wave equation. J. Funct. Anal., 110(1):96-130, 1992.

[14] J. Ginibre and G. Velo. Generalized Strichartz inequalities for the wave equation. J. Funct. Anal., 133(1):50-68, 1995.

[15] R. T. Glassey. On the blowing up of solutions to the Cauchy problem for nonlinear Schrödinger equations. J. Math. Phys., 18(9):1794-1797, 1977.

[16] M. G. Grillakis. Regularity and asymptotic behaviour of the wave equation with a critical nonlinearity. Ann. of Math. (2), 132(3):485-509, 1990.

[17] M. G. Grillakis. Regularity for the wave equation with a critical nonlinearity. Comm. Pure Appl. Math., 45(6):749-774, 1992.

[18] M. G. Grillakis. On nonlinear Schrödinger equations. Comm. Partial Differential Equations, 25(9-10):1827-1844, 2000.

[19] L. Hörmander. The analysis of linear partial differential operators. III, volume 274 of Grundlehren der Mathematischen Wissenschaften [Fundamental Principles of Mathematical Sciences]. Springer-Verlag, Berlin, 1985. Pseudodifferential operators.

[20] L. Kapitanski. Global and unique weak solutions of nonlinear wave equations. Math. Res. Lett., 1(2):211-223, 1994.

[21] M. Keel and T. Tao. Endpoint Strichartz estimates. Amer. J. Math., 120(5):955-980, 1998.

[22] C. Kenig. Global well-posedness and scattering for the energy critical focusing non-linear Schrödinger and wave equations. Lecture Notes for a minicourse given at "Analyse des équations aux derivées partialles", Evian-lesbains, June 2007.

[23] C. Kenig and F. Merle. Global well-posedness, scattering and blow-up for the energy critical focusing non-linear wave equation. Acta Math., to appear.

[24] C. Kenig and F. Merle. Scattering for $\dot{H}^{1 / 2}$ bounded solutions to the cubic defocusing NLS in 3 dimensions. Trans. Amer. Math. Soc., to appear.

[25] C. Kenig and F. Merle. Global well-posedness, scattering and blow-up for the energy-critical, focusing, non-linear Schrödinger equation in the radial case. Invent. Math., 166(3):645-675, 2006. 
[26] C. Kenig, G. Ponce, and L. Vega. Well-posedness and scattering results for the generalized Korteweg-de Vries equation via the contraction principle. Comm. Pure Appl. Math., 46(4):527-620, 1993.

[27] S. Keraani. On the defect of compactness for the Strichartz estimates of the Schrödinger equations. J. Differential Equations, 175(2):353-392, 2001.

[28] R. Killip, T. Tao, and M. Vişan. The cubic nonlinear Schrödinger equation in two dimensions with radial data, 2007. arXiv:math.AP/0707.3188.

[29] R. Killip and M. Vişan. The focusing energy-critical nonlinear Schrödinger equation in dimensions five and higher, 2008. arXiv:math.AP/0804.1018.

[30] R. Killip, M. Vişan, and X. Zhang. The mass-critical nonlinear Schrödinger equation with radial data in dimensions three and higher, 2007. arXiv:math.AP/0708.0849.

[31] J. Krieger, W. Schlag, and D. Tătaru. Renormalization and blow up for charge and equivariant critical wave maps. Invent. Math., to appear.

[32] J. Krieger, W. Schlag, and D. Tătaru. Slow blow-up solutions for the $H^{1}\left(\mathbb{R}^{3}\right)$ critical focusing semi-linear wave equation in $\mathbb{R}^{3}, 2007$. Preprint, arXiv:math.AP/0711.1818.

[33] H. Levine. Instability and nonexistence of global solutions to nonlinear wave equations of the form $P u_{t t}=-A u+\mathcal{F}(u)$. Trans. Amer. Math. Soc., 192:121, 1974.

[34] H. Lindblad and C. Sogge. On existence and scattering with minimal regularity for semilinear wave equations. J. Funct. Anal., 130(2):357-426, 1995.

[35] F. Merle and L. Vega. Compactness at blow-up time for $L^{2}$ solutions of the critical nonlinear Schrödinger equation in 2D. Internat. Math. Res. Notices, (8):399-425, 1998.

[36] F. Merle and H. Zaag. Determination of the blow-up rate for the semilinear wave equation. Amer. J. Math., 125(5):1147-1164, 2003.

[37] H. Pecher. Nonlinear small data scattering for the wave and Klein-Gordon equation. Math. Z., 185(2):261-270, 1984.

[38] P. Raphaël. Existence and stability of a solution blowing up on a sphere for an $L^{2}$-supercritical nonlinear Schrödinger equation. Duke Math. J., 134(2):199$258,2006$.

[39] I. Rodnianski and J. Sterbenz. On the formation of singularities in the critical $O(3)$ sigma model. Annals of Math., to appear. 
[40] E. Ryckman and M. Vişan. Global well-posedness and scattering for the defocusing energy-critical nonlinear Schrödinger equation in $\mathbb{R}^{1+4}$. Amer. J. Math., 129(1):1-60, 2007.

[41] J. Shatah and M. Struwe. Well-posedness in the energy space for semilinear wave equations with critical growth. Internat. Math. Res. Notices, (7):303ff., approx. 7 pp. (electronic), 1994.

[42] J. Shatah and M. Struwe. Geometric wave equations, volume 2 of Courant Lecture Notes in Mathematics. New York University Courant Institute of Mathematical Sciences, New York, 1998.

[43] R. Strichartz. Restrictions of Fourier transforms to quadratic surfaces and decay of solutions of wave equations. Duke Math. J., 44(3):705-714, 1977.

[44] M. Struwe. Globally regular solutions to the $u^{5}$ Klein-Gordon equation. Ann. Scuola Norm. Sup. Pisa Cl. Sci. (4), 15(3):495-513 (1989), 1988.

[45] M. Struwe. Equivariant wave maps in two space dimensions. Comm. Pure Appl. Math., 56(7):815-823, 2003. Dedicated to the memory of Jürgen Moser.

[46] G. Talenti. Best constant in Sobolev inequality. Ann. Mat. Pura Appl. (4), 110:353-372, 1976.

[47] T. Tao. Global regularity of wave maps. II. Small energy in two dimensions. Comm. Math. Phys., 224(2):443-544, 2001.

[48] T. Tao. Global well-posedness and scattering for the higher-dimensional energy-critical nonlinear Schrödinger equation for radial data. New York J. Math., 11:57-80 (electronic), 2005.

[49] T. Tao and M. Vişan. Stability of energy-critical nonlinear Schrödinger equations in high dimensions. Electron. J. Differential Equations, pages No. 118, 28 pp. (electronic), 2005.

[50] T. Tao, M. Vişan, and X. Zhang. Global well-posedness and scattering for the defocusing mass-critical nonlinear Schrödinger equation for radial data in high dimensions. Duke Math. J., 140(1):165-202, 2007.

[51] N. Trudinger. Remarks concerning the conformal deformation of Riemannian structures on compact manifolds. Ann. Scuola Norm. Sup. Pisa (3), 22:265$274,1968$.

[52] D. Tătaru. On global existence and scattering for the wave maps equation. Amer. J. Math., 123(1):37-77, 2001.

[53] D. Tătaru. Rough solutions for the wave maps equation. Amer. J. Math., $127(2): 293-377,2005$. 
[54] M. Vişan. The defocusing energy-critical nonlinear Schrödinger equation in higher dimensions. Duke Math. J., 138(2):281-374, 2007. 